\title{
Biomaterials
}

\section{Poly(E-caprolactone)-based membranes with tunable physicochemical, bioactive and osteoinductive properties}

\author{
Michal Dziadek ${ }^{1, *}$ (1), Barbara Zagrajczuk ${ }^{1}$ (D), Elzbieta Menaszek $^{2}$ (D), Kinga Dziadek ${ }^{3}$ (1), \\ and Katarzyna Cholewa-Kowalska ${ }^{1}$ (D) \\ ${ }^{1}$ Department of Glass Technology and Amorphous Coatings, Faculty of Materials Science and Ceramics, AGH University of Science \\ and Technology, 30 Mickiewicza Ave., 30-059 Kraków, Poland \\ ${ }^{2}$ Department of Cytobiology, Collegium Medicum, Jagiellonian University, 9 Medyczna St., 30-688 Kraków, Poland \\ ${ }^{3}$ Department of Human Nutrition, Faculty of Food Technology, University of Agriculture in Krakow, 122 Balicka St., 30-149 Kraków, \\ Poland
}

Received: 16 March 2017

Accepted: 24 July 2017

Published online:

3 August 2017

(C) The Author(s) 2017. This article is an open access publication

\section{ABSTRACT}

In contrast to currently used materials, membranes for the treatment of bone defects should actively promote regeneration of bone tissue beyond their physical barrier function. What is more, both material properties and biological features of membranes should be easily adaptable to meet the needs of particular therapeutic applications. Therefore, the role of preparation methods (nonsolvent-induced phase separation and thermal-induced phase separation) of poly( $\varepsilon$-caprolactone)-based membranes and their modification with gel-derived bioactive glass (BG) particles of two different sizes $(<45$ and $<3 \mu \mathrm{m})$ in modulating material morphology, polymer matrix crystallinity, surface wettability, kinetics of in vitro bioactivity and also osteoblast response was investigated. Both surfaces of membranes were characterised in terms of their properties. Our results indicated a possibility to modulate microstructure (pore size ranging from submicron to hundreds of micrometres), wettability (from hydrophobic to fully wettable surface) and polymer crystallinity (from 19 to 60\%) in a wide range by the use of various preparation methods and different BG particle sizes. Obtained composite membranes showed excellent in vitro hydroxyapatite forming ability after incubation in simulated body fluid. Here we demonstrated that bioactive layer formation on the surface of membranes occurred through ACP-OCP-CDHA-HCA transformation, that mimic in vivo bone biomineralization process. Composite membranes supported human osteoblast proliferation, stimulated cell differentiation and matrix mineralization. We proved that kinetics of bioactivity process and also osteoinductive properties of membranes can be easily modulated with the use of proposed variables. This brings new

Address correspondence to E-mail: dziadek@agh.edu.pl 
opportunities to obtain multifunctional membranes for bone regeneration with tunable physicochemical and biological properties.

\section{Introduction}

Tissue engineering (TE) offers a promising approach to repair damaged tissues and/or to promote new tissue growth using highly porous biomaterials, cells and signalling molecules [1]. Biomaterials for TE, depending on their target applications, are prepared in the form of three-dimensional scaffolds or porous membranes. Scaffolds act as temporary, artificial extracellular matrix (ECM) for cell attachment, proliferation, migration, differentiation and create a unique environment that enables and/or induces tissue regeneration [1, 2]. In turn, porous membranes are mainly used for guided tissue/bone regeneration (GTR/GBR), to prevent connective tissue ingrowth into the bone defects and to maintain a suitable space for bone regeneration processes [3, 4]. Another application for porous membranes is at the interfaces between soft and hard tissues, e.g. cartilage/bone interface [5]. Porous biomaterials for TE have to meet several requirements: (1) uniformly distributed and interconnected highly porous structure with suitable pore size and shape to provide adequate space for cells seeding or growth, blood vessel ingrowth and flow transport of nutrients and metabolic waste; (2) biodegradable or bioresorbable with a controllable degradation and resorption rate, appropriate to match tissue growth in vivo and with non-cytotoxic degradation products; (3) suitable surface topography, chemistry and wettability for cell attachment, proliferation and differentiation; (4) mechanical properties matching those of the tissues at the site of implantation $[2,6,7]$. Furthermore, materials for bone tissue engineering (BTE) should possess bone-bonding ability, as well as osteoconductive/osteoinductive properties $[8,9]$.

Phase separation methods, predominantly the nonsolvent-induced phase separation (NIPS, also called as immersion-precipitation) $[3,10,11]$ and the thermalinduced phase separation (TIPS) [10, 12-15] techniques have been used to obtain porous polymer, as well as polymer-ceramic composite scaffolds and membranes for TE applications. The obtained material morphology, as well as the corresponding pore size and shape may vary widely depending on the manufacturing process conditions; from dense to highly porous, and with pores size from submicron scale to tens of microns. The NIPS method includes three components (polymer, solvent and non-solvent). The polymer solution is cast on a glass support and immersed in a coagulation (non-solvent) bath. The phase separation occurs because of the exchange of solvent and nonsolvent. Many parameters such as polymer crystallinity, polymer concentration in the solution, temperature of the casting solution and of the coagulation bath, type of solvent and non-solvent and their mutual affinity affect phase separation process and consequently the final morphology and properties of porous materials $[11,16]$. On the other hand, TIPS method is composed of only two components (polymer and solvent). The polymer solution is cast onto a plate cooled to the temperature well below the solvent freezing point. Solvent crystallization (solid-liquid separation) occurs, and polymer is separated from the solvent crystallization front. Solvent crystals, after removing by freeze-drying or extraction, provide pores in the material. TIPS is a versatile processing method allowing considerable control over membrane and scaffold microstructure. Adjusting the parameters of the TIPS process, such as polymer type, polymer concentration, quenching temperature and time, and the cooling rate, can lead to porous polymer and composite constructs with distinctive architectures, tailored to the target applications [14, 15, 17].

Besides the various preparation methods, incorporation of the inorganic phase (e.g. bioactive glasses, glass-ceramics, silica, wollastonite, calcium phosphates) in biodegradable polymer matrix is another strategy for modulating a number of properties of porous materials for TE [3, 8]. Such approach is particularly suitable for BTE applications because of the polymer/ceramic composites ability to mimic the structure of natural bone [9].

Poly( $\varepsilon$-caprolactone) (PCL)-based composites reinforced with bioactive glass particles have been drawing increasing interest as materials for BTE applications $[9,18]$. Such composites combine excellent biocompatibility, relatively high mechanical strength, ease of processing, as well as low degradation rate of PCL [9, 18-20] with excellent bone-bonding 
ability, osteoconductive and/or osteoinductive character, stiffness and high hydrophilicity of bioactive glasses $[8,9,18,21,22]$. Furthermore, there is a possibility to use sol-gel-derived glass particles that have a larger surface area and $-\mathrm{OH}$ groups present in their structure, and therefore usually exhibit higher bioactivity than conventional melt-derived glasses [23, 24].

Based on the facts discussed above, in the present investigation, PCL-based porous membranes were obtained using two phase separation methods: the non-solvent-induced phase separation (NIPS) and the thermal-induced phase separation (TIPS). Materials were modified with gel-derived $\mathrm{SiO}_{2}-\mathrm{CaO}-\mathrm{P}_{2} \mathrm{O}_{5}$ bioactive glass particles of two different sizes: $<3$ and $<45 \mu \mathrm{m}$. The effect of these variables on surface morphology and wettability, as well as polymer matrix crystallinity was studied. Depending on glass particle size and membrane preparation method, kinetics of in vitro apatite formation, as well as response of normal human osteoblasts, including cell proliferation, alkaline phosphatase activity (ALP) and matrix mineralization, were investigated.

\section{Materials and methods}

\section{Materials}

Poly(e-caprolactone) (PCL; Mn 80 kDa, Mw/Mn < 2; Sigma-Aldrich, USA) and A2 bioactive glass particles were used as composite components. Bioactive glass (SBG) of the following composition $(\mathrm{mol} \%) 40 \mathrm{SiO}_{2}-$ $54 \mathrm{CaO}-6 \mathrm{P}_{2} \mathrm{O}_{5}$ was produced using the sol-gel method described in our previous work [25]. In order to prepare composite membranes, two different solvents were used: 1,4-dioxane (DIOX) and $\mathrm{N}, \mathrm{N}-$ dimethylformamide (DMF) (POCh, Poland).

\section{Bioactive glass preparation}

Tetraethoxysilane (TEOS; $\left.\mathrm{Si}\left(\mathrm{OC}_{2} \mathrm{H}_{5}\right)_{4}\right)$, triethylphosphate (TEP; $\left.\mathrm{OP}\left(\mathrm{OC}_{2} \mathrm{H}_{5}\right)_{3}\right)$ (Sigma-Aldrich, USA) and calcium nitrate tetrahydrate $\left(\mathrm{Ca}\left(\mathrm{NO}_{3}\right)_{2} \cdot 4 \mathrm{H}_{2} \mathrm{O}\right)(\mathrm{POCh}$, Poland) were used as basic components of the sol-gel process. $1 \mathrm{M} \mathrm{HCl}$ solution (POCh, Poland) was used as a catalyst in the hydrolysis and condensation reactions. The molar ratios of TEOS:TEP:Ca $\left(\mathrm{NO}_{3}\right)_{2}$ : $\mathrm{H}_{2} \mathrm{O}: \mathrm{HCl}$ were $1: 0.3: 1.4: 4: 0.2$. Formed gel was gradually dried at the temperature increasing in the range from 40 to $120^{\circ} \mathrm{C}$ for 7 days and then subjected to the thermal treatment at $700{ }^{\circ} \mathrm{C}$ for $20 \mathrm{~h}$. Two glass particle sizes were obtained: $<45 \mu \mathrm{m}$ by grinding and sieving and $<3 \mu \mathrm{m}$ by milling in an attritor with $\mathrm{ZrO}_{2}$ grinding balls in isopropyl alcohol medium. Particle size distributions in powder aqueous suspensions were analysed using laser diffraction method (Mastersizer 2000, Malvern, UK).

\section{Composite membrane preparation}

The PCL-bioactive glass membranes were fabricated with two methods: thermal-induced phase separation (TIPS) and non-solvent-induced phase separation (NIPS). Polymer-bioactive glass suspensions were prepared by mixing glass particles with $5 \mathrm{w} / \mathrm{v} \%$ PCL solutions in DIOX (for TIPS) and DMF (for NIPS), on a magnetic stirrer for $24 \mathrm{~h}$.

Thermal-induced phase separation The suspensions were cast onto glass Petri dishes (diameter $90 \mathrm{~mm}$ ), covered with glass Petri dish lids (diameter $100 \mathrm{~mm}$ ) and placed in a laboratory freezer at the temperature of $-80{ }^{\circ} \mathrm{C}$ for $24 \mathrm{~h}$. Afterwards, freeze-drying process was performed using freeze-dry system (FreeZone $6 \mathrm{l}$, Labconco, USA) for $48 \mathrm{~h}$.

Non-solvent-induced phase separation The suspensions, heated to $60^{\circ} \mathrm{C}$, were cast onto preheated $\left(60^{\circ} \mathrm{C}\right)$ glass plate using a custom-made doctor blade with a $250-\mu \mathrm{m}$ gap to obtain a uniform polymer solution film. Immediately after casting, the glass plate with suspension was immersed in distilled $\mathrm{H}_{2} \mathrm{O}$ at the temperature of $10{ }^{\circ} \mathrm{C}$ for $10 \mathrm{~min}$. Afterwards, membranes were thoroughly rinsed with distilled $\mathrm{H}_{2} \mathrm{O}$ and dried under ambient conditions to constant weight.

The reference membranes (pure PCL) were prepared with the use of PCL solutions in appropriate solvents applying the same methods as mentioned above. The resulting membranes were examined with consideration for the differences between the specimen surfaces. The surface that had been in contact with glass Petri dish or glass plate during casting was marked as GS, while the other one was marked as AS.

\section{Material evaluation}

\section{SEM/EDX analysis}

Surface morphology and chemical composition were determined using scanning electron microscopy 
(SEM, Nova NanoSEM 200 FEI Europe Company) coupled with energy dispersion X-ray (EDX) analyser. The SEM evaluation was also used for estimation of surface pore size and shape. The samples were covered with a carbon layer.

\section{Apparent water contact angle measurements}

Surface wettability was evaluated by the apparent water contact angle measurements. The contact angle was determined by sessile drop method with an automatic drop shape analysis system DSA $10 \mathrm{Mk} 2$ (Kruss, Germany). UHQ-water droplets of $0.25 \mu \mathrm{L}$ were applied on every, pure and dry, sample. Measurements were carried out at constant conditions (temperature and humidity). The apparent contact angle was calculated as an average of ten measurements and was expressed as mean \pm standard deviation (SD). Furthermore, the evolution of the contact angle over time was registered.

\section{DSC analysis}

Degree of crystallinity and melting temperature of PCL were measured with power compensation differential scanning calorimetry (DSC, PerkinElmer DSC-7, USA). The melting temperature $\left(T_{\mathrm{m}}\right)$ was determined at the maximum of the melting endotherm during a single heating run. The degree of crystallinity $\left(\chi_{c}\right)$ was estimated using the enthalpy of melting change according to the equation: $\chi_{\mathrm{c}}=\Delta H_{\mathrm{m}} /(1-x) \Delta H_{\mathrm{m}}^{0}$, where $\Delta H_{\mathrm{m}}$ and $\Delta H_{\mathrm{m}}^{0}$ were the enthalpy of melting of the sample and of fully crystalline PCL (139.5 $\mathrm{J} \mathrm{g}^{-1}$ ) [20], respectively, and $x$ was the weight fraction of the bioactive glass particles. All of the samples (average weight $10 \mathrm{mg}$ ) were placed in standard aluminium pans. The specimens were scanned from 20 to $100{ }^{\circ} \mathrm{C}$ with the heating rate of $10{ }^{\circ} \mathrm{C} \mathrm{min}{ }^{-1}$, using nitrogen as a purge gas. The results were average from the three measurements and were expressed as mean $\pm \mathrm{SD}$.

\section{In vitro bioactivity test}

In vitro bioactivity was evaluated by incubation of materials in simulated body fluid (SBF) prepared according to Kokubo [26]. The samples were immersed in SBF solution and incubated at $37{ }^{\circ} \mathrm{C}$ in separate polypropylene containers for 3, 7 and 14 days. The sample weight to SBF volume ratio was
$10^{-3} \mathrm{~g} \mathrm{ml}^{-1}$. Afterwards, the samples were washed with anhydrous ethanol and air-dried at room temperature to a constant weight. Both surfaces of each sample were examined with SEM/EDX (Nova NanoSEM 200 FEI Europe Company) and ATR-FTIR (Bruker VERTEX $70 \mathrm{~V}$ spectrometer, USA) methods.

$\mathrm{Ca} / \mathrm{P}$ molar ratios of the layers formed on GS surfaces of the membranes were calculated based on a semi-quantitative analyses of the EDX spectra collected from at least three different points of each sample. The ATR-FTIR spectra were registered with the use of a platinum single crystal diamond ATR unit in the $550-4000 \mathrm{~cm}^{-1}$ wavenumber range, and 128 scans were accumulated at $4 \mathrm{~cm}^{-1}$ resolution. Furthermore, the changes in the concentration of calcium, phosphorus and silicon in the SBF during membrane incubation were analysed for each testing time using inductively coupled plasma atomic emission spectrometry (ICP-OES; Plasm 40, Perkin Elmer, USA). The test was performed in triplicate and expressed as mean $\pm \mathrm{SD}$.

In vitro osteoblast response

Material sterilization For the cell culture, membranes (round samples matching the size of wells of 48-well culture plate) were sterilized by soaking in $70 \%$ ethanol for $30 \mathrm{~min}$, further both sides of samples were sterilized with UV-C light for $30 \mathrm{~min}$ each and washed with sterile phosphate-buffered saline (PBS, HyClone, USA).

Cell culture The normal human osteoblasts (NHOst, Lonza, USA) were expanded in $75 \mathrm{~cm}^{2}$ tissue culture flasks (Nunc ${ }^{\mathrm{TM}}$, Denmark) in complete osteoblast growth medium OGM BulletKit (Lonza, USA) containing $10 \%$ FBS, $0.1 \%$ ascorbic acid and $0.1 \%$ GA1000 (gentamicin sulphate and amphotericin-B) at $37^{\circ} \mathrm{C}$ in a humidified, $5 \% \mathrm{CO}_{2}$ atmosphere. Medium was changed every 3 days until a $70 \%$ confluent cell monolayer had developed. Then cells were detached from culture flasks using 5\% Trypsin-EDTA (HyClone, USA).

The prepared sterile membranes were placed at a bottom of 48-well culture plate wells (Nunc ${ }^{\mathrm{TM}}$, Denmark) and held by ultrapure silica glass inserts to prevent samples floating. NHOst cells were seeded on the GS surface of membranes at a density of $1.5 \times 10^{4}$ cells $/ \mathrm{mL} /$ well and cultured for 7,14 and 21 days in complete osteoblast growth medium OGM 
supplemented with differentiation kit SingleQuots (Lonza, USA), containing hydrocortisone-21hemisuccinate and $\beta$-glycerophosphate. The bottom surfaces of tissue culture polystyrene (TCPS) wells served as a control.

Microscopic observation After 21 days of culture, membranes were rinsed with PBS and then cells were fixed with $3 \%$ glutaraldehyde solution in sodium cacodylate buffer, $\mathrm{pH} 7.4$ (POCh, Poland) for $1 \mathrm{~h}$. Subsequently, the cells were dehydrated in graded series of ethanol solution (70, 80, 90, 96 and 100\%) and dried in air. Cell morphologies were evaluated using SEM (Nova NanoSEM 200 FEI Europe Company) after coating with carbon.

After 14 days of culture, the cells were stained with $0.01 \%$ acridine orange $(\mathrm{AO})$ solution (Sigma-Aldrich, USA) for $1 \mathrm{~min}$. Next, the samples were rinsed with PBS, observed, and photographed under the fluorescence microscope (Olympus CX41, Japan).

Cell proliferation and material cytotoxicity In order to determine proliferation rate of NHOst cells and cytotoxic effect of obtained materials, ToxiLight ${ }^{\mathrm{TM}}$ BioAssay Kit and ToxiLight ${ }^{\mathrm{TM}}$ 100\% Lysis Reagent Set (Lonza, USA) were used according to manufacturer's protocol. The kit was used to quantify adenylate kinase (AK) in both supernatant (representing damaged cells) and lysate (representing intact adherent cells). The results were expressed as mean \pm SD from eight samples for each experimental group.

Alkaline phosphatase activity Alkaline phosphatase (ALP) activity measurement is based on the hydrolysis reaction of 4-MUP (4-methylumbelliferyl phosphate, the substrate for ALP expressed by differentiated osteoblasts), to highly fluorescent product 4-MU (4-methylumbelliferone). After 7 days of culture, NHOst cells were disrupted via a cyclic freezing/thawing in order to release intracellular ALP. Cell lysates in triplicates were transferred to OptiPlate-96 microplate (PerkinElmer) and incubated with an equal volumes of 4-MUP Liquid Substrate System (Sigma-Aldrich, USA) solution for $1 \mathrm{~h}$. Fluorescence was determined at 360/440 nm (excitation/ emission wavelengths) using POLARstar Omega microplate reader (BMG Labtech, Germany). The results were expressed as mean \pm SD from eight samples for each experimental group.
ECM mineralization Extracellular matrix (ECM) mineralization in cell culture was analysed using fluorescent OsteoImage ${ }^{\mathrm{TM}}$ Mineralization Assay (Lonza, USA) after 14 and 21 days of culture according to manufacturer's protocol. The results were expressed as mean \pm SD from four samples for each experimental group.

\section{Statistical analysis}

The results were analysed using one-way analysis of variance (ANOVA) with Duncan post hoc tests, which were performed with Statistica 10 (StatSoft ${ }^{\mathbb{B}}$, USA) software. The results were considered statistically significant when $p<0.05$.

\section{Results}

\section{Surface morphology}

Figure 1 shows the morphologies of both AS and GS surfaces of the membranes obtained with the NIPS and TIPS methods. Evident differences between AS and GS surfaces of obtained polymer and composite membranes were found. AS surfaces of materials prepared by TIPS method exhibited open, irregular, cellular microporous structure, a typical morphology formed by solid-liquid phase separation [12-14]. Pore size decreased, and their shape become more regular upon addition of BG particles. However, particle size did not show obvious effect on the overall membrane porosity. Smaller glass particles $(<3 \mu \mathrm{m})$ were evident on the AS surfaces of membranes, confirmed by EDX analysis ( $\mathrm{Si}, \mathrm{Ca}$ and $\mathrm{P}$ derived from the glass were detected), while largersized ones $(<45 \mu \mathrm{m})$ were not detected. GS surface of the PCL/TIPS membrane showed elongated pores oriented in one direction. The addition of BG particles significantly reduced porosity of the GS surface of PCL/TIPS membrane. GS surface of PCL/ A2 $<45 \mu \mathrm{m} /$ TIPS was mainly consisted of glass particles, which was also confirmed by EDX analysis, indicating intensive sedimentation of $<45 \mu \mathrm{m}$ BG particles in polymer solution during preparation process.

Membranes prepared with the use of NIPS method showed high porosity of both surfaces, without dense nonporous top layer, characteristic for some membranes produced using this method [27]. AS surfaces 


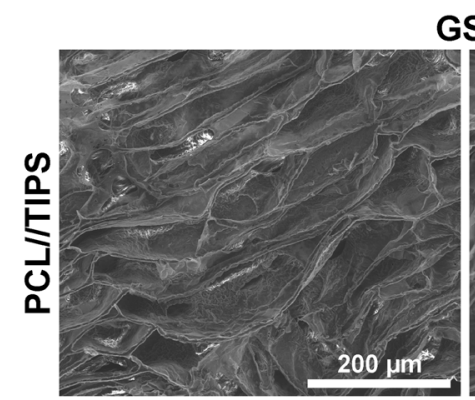

GS
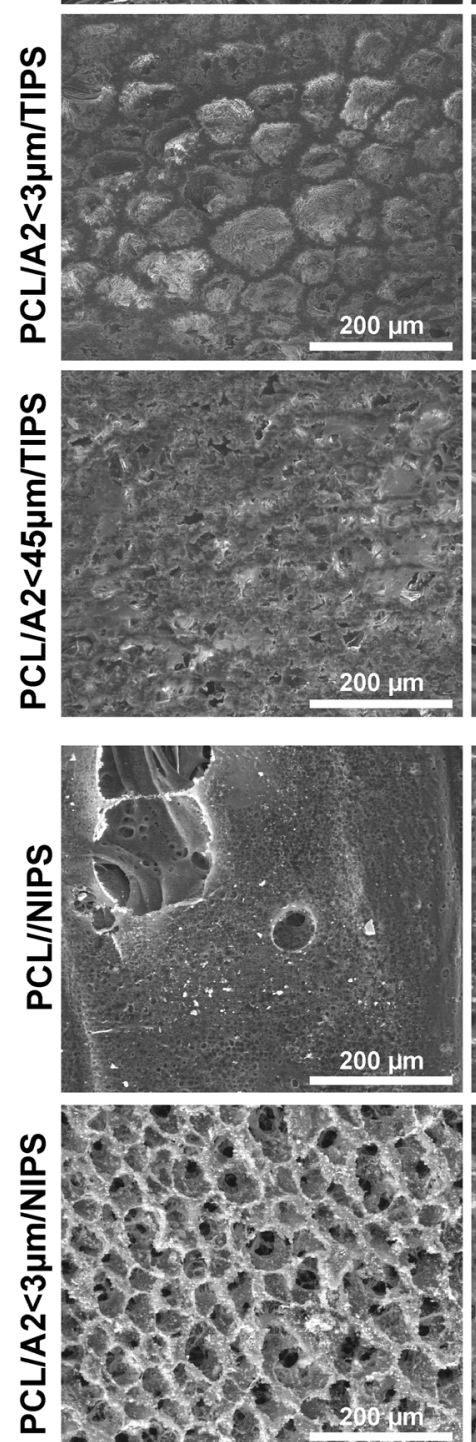

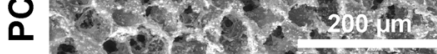

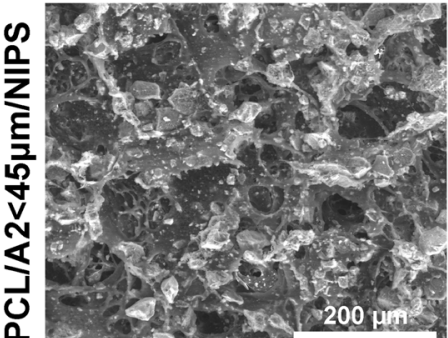

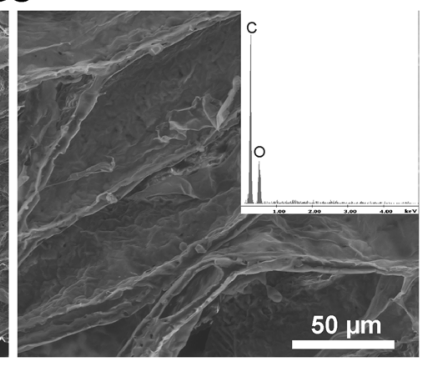
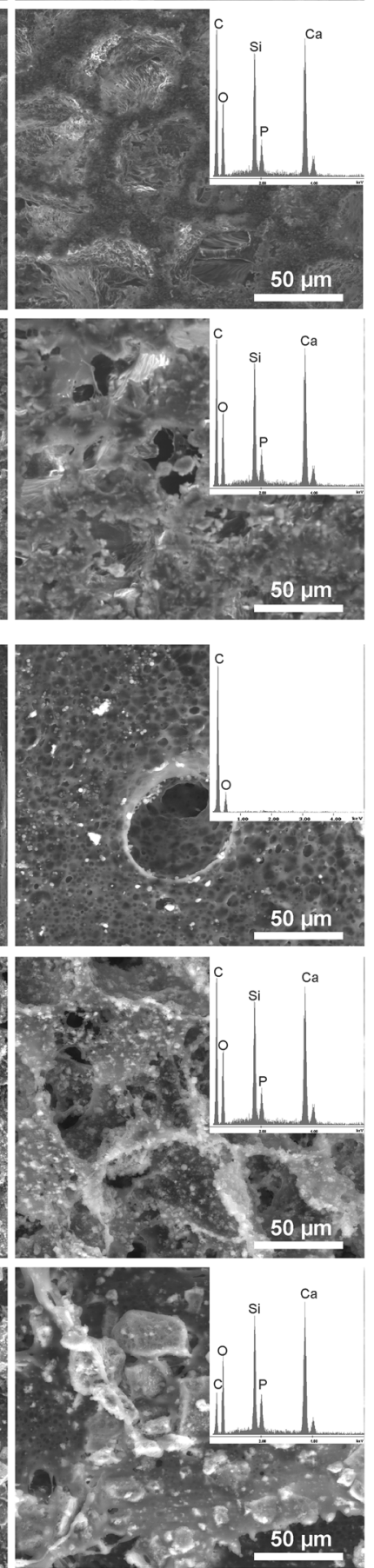

AS
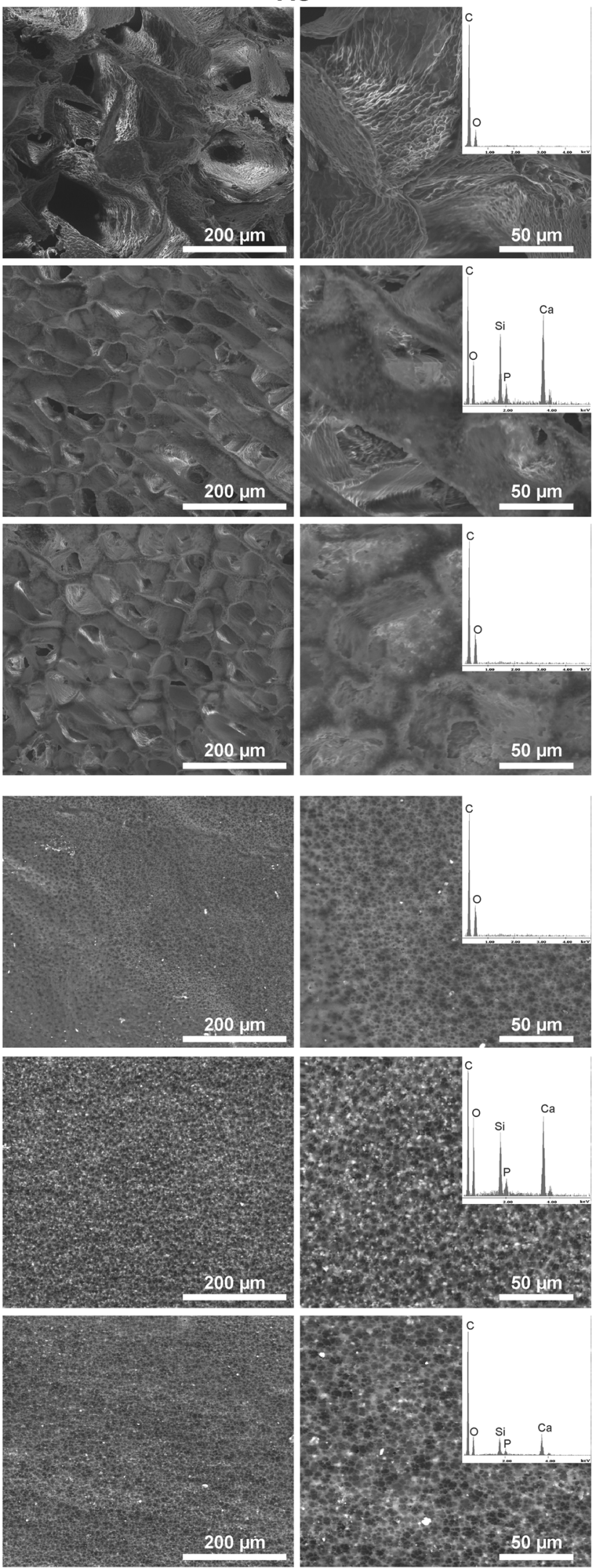

$50 \mathrm{um}$

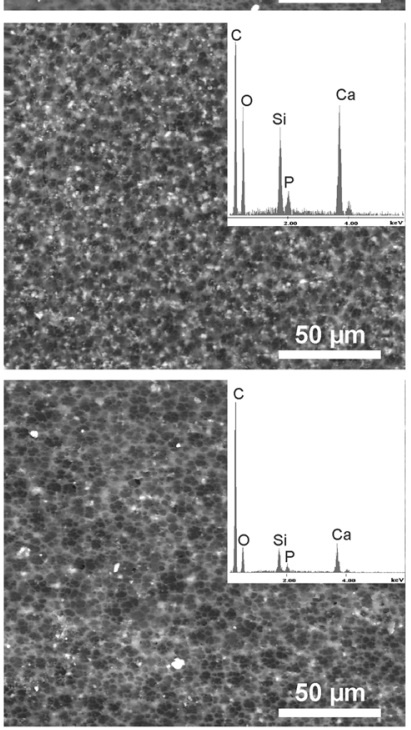


4 Figure 1 SEM images and EDX spectra (averaged for the entire analysed surface) of both GS and AS surfaces of membranes obtained with the use of TIPS and NIPS methods.

of membranes prepared by NIPS technique showed uniformly distributed submicron and micron-sized pores, as it was observed using SEM. The addition of BG particles of both sizes did not change AS surface microstructure significantly. The only difference was that AS surface of the PCL/A2 $<3 \mu \mathrm{m} / \mathrm{NIPS}$ membrane contained greater amount of glass particles compared to the same surface of the PCL/ A2 $<45 \mu \mathrm{m} /$ NIPS material, which was also confirmed by EDX analysis. The presence of fraction of smaller particles on the AS surface of the PCL/ A $2<45 \mu \mathrm{m} /$ NIPS membrane indicates higher rate of membrane formation in NIPS method reducing sedimentation of BG particles in polymer solution. GS surface of the PCL/NIPS membrane consisted of two different pore size ranges. Large pores, sized from tens to hundreds of micrometres, and much smaller submicron and micron-sized pores (similar to that on AS surface) in the walls of the large pores and on the membrane surface were developed. When BG particles were introduced into the PCL/NIPS matrix, an open and regular pores were formed with an average pore size of $50-80 \mu \mathrm{m}$ and $150-200 \mu \mathrm{m}$ on the GS surface of the PCL/A2 $<3 \mu \mathrm{m} / \mathrm{NIPS}$ and PCL/ A2 $<45 \mu \mathrm{m} /$ NIPS membranes, respectively. BG particles were uniformly distributed within the pore walls of the membranes; however, the overall porosity of the PCL/A2 $<45 \mu \mathrm{m} /$ LIPS seemed to be slightly lower compared to material with smaller BG particles.

\section{Static water contact angle}

The average values of static water contact angle measured immediately after the water drop application on both GS and AS surfaces of obtained membranes are shown in Fig. 2a. In case of membranes prepared with TIPS method, GS surfaces of the PCL/TIPS and PCL/ A2 $<3 \mu \mathrm{m} /$ TIPS materials showed significantly lower values compared to AS surfaces, while between both surfaces of the PCL/A2 $<45 \mu \mathrm{m} / \mathrm{TIPS}$ composite there was no significant difference in the contact angle value. The addition of BG particles of $<3 \mu \mathrm{m}$ size into the PCL/TIPS matrix resulted in reduction in contact angle of both AS and GS surfaces, while larger-sized particles $(<45 \mu \mathrm{m})$ led to a decrease only for AS surface. When considering membranes obtained with the use of NIPS technique, wettability was significantly improved with respect to the polymer and composite PCL/TIPS membranes, which is also showed in Fig. $2 b$, where contact angle changes over time are presented. The polymer PCL/NIPS membrane showed opposite trend to the PCL/TIPS material, namely GS surface exhibited significantly higher contact angle values compared to AS surface. As in case of the PCL/A2 $<3 \mu \mathrm{m} /$ TIPS membrane, GS surfaces of both composites obtained with NIPS method showed significantly improved wettability compared to AS surface. In particular, when the water drop contacted GS surface of the PCL/A2 $<3 \mu \mathrm{m} /$ NIPS membrane, complete wetting occurred, therefore that contact angle could not have been measured. The incorporation of BG particles of $<3 \mu \mathrm{m}$ size into the PCL/NIPS matrix resulted in reduction in contact angle of both AS and GS surfaces. Particles of $<45 \mu \mathrm{m}$ size influenced both surfaces of the PCL/NIPS membrane in an opposite manner: contact angle of GS surface was reduced, while AS surface tends to be more hydrophobic compared to the surfaces of polymer PCL/NIPS membrane, but yet both surfaces were more hydrophilic than the PCL/A2 $<45 \mu \mathrm{m} / \mathrm{TIPS}$ material.

The changes in water contact angle over time confirmed that the membranes obtained with NIPS method were more hydrophilic compared to the group of TIPS materials (Fig. 2b). What is more, contact angle of these latter membranes did not change significantly over time, regardless of tested surface. Similar behaviour showed polymer PCL/ NIPS material. In case of the PCL/A2 $<3 \mu \mathrm{m} / \mathrm{NIPS}$ and PCL/A2 $<45 \mu \mathrm{m} / \mathrm{NIPS}$ membranes, spreading/ penetration of a water droplet over time occurred. This suggests that modification of the PCL/NIPS membrane with BG particles affected wettability more effectively compared to materials produced with TIPS method. Furthermore, the rate of droplet spreading on GS surface of the PCL/A2 $<45 \mu \mathrm{m} /$ NIPS composite was noticeably higher compared to AS surface. These results indicate that GS surface of composite obtained with NIPS technique was more hydrophilic. Even though the AS surface of the PCL/ A2 $<45 \mu \mathrm{m} /$ NIPS material exhibited significantly higher contact angle compared to the same surface of the PCL/NIPS membrane, droplet spreading assessment showed that this surface was more wettable. 


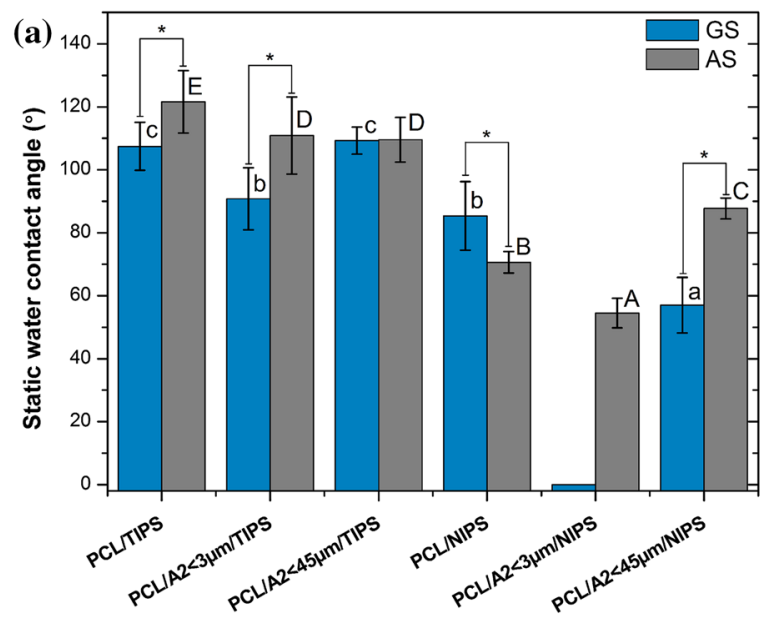

(b)
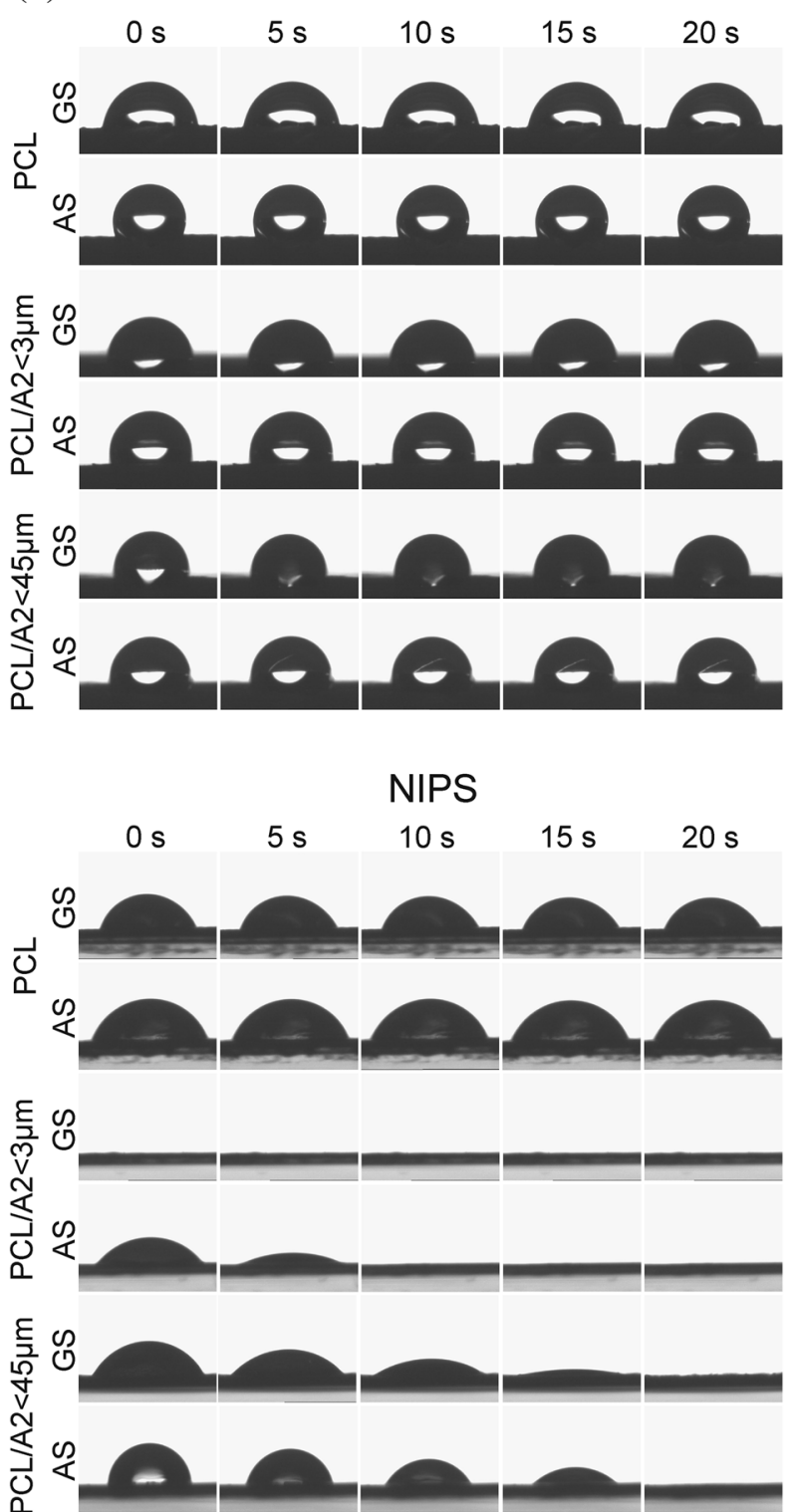

4 Figure 2 Average values of static water contact angle measured immediately after application of the water drop on the both GS and AS surfaces of obtained membranes (a) and the changes in water contact angle over time (b). Results are expressed as mean \pm SD. Statistically significant differences $(p<0.05)$ : between membranes are indicated by lower (for GS surface) and upper (for AS surface) case; between the GS and AS surfaces of the same material are indicated by asterisk $(*)$.

\section{DSC analysis}

The results of the melting temperature $\left(T_{\mathrm{m}}\right)$ and the degree of crystallinity $\left(\chi_{c}\right)$ of obtained membranes estimated according to DSC analysis are shown in Table 1. It was found that degree of crystallinity of membranes prepared with the use of NIPS method was lower in comparison with values recorded for materials obtained with TIPS technique. In particular, $\chi_{c}$ value of the PCL/NIPS membrane $(29.19 \%)$ was a half of that for the PCL/TIPS material $(60.25 \%)$. Furthermore, the PCL/NIPS material showed significantly lower melting temperature $\left(53.33^{\circ} \mathrm{C}\right)$ compared to the PCL/TIPS membrane $\left(59.17{ }^{\circ} \mathrm{C}\right)$, implying smaller/thinner PCL crystallites/lamellae and/or the decrease in crystalline perfection [20,28].

\section{In vitro bioactivity}

\section{SEM/EDX analysis}

Obtained membranes were examined in terms of in vitro bioactivity by soaking them in SBF for 3, 7 and 14 days. Figures 3 and 4 show SEM images and EDX spectra of GS and AS surfaces of the membranes obtained with the use of TIPS and NIPS techniques, respectively, after incubation in SBF. The PCL/NIPS and PCL/TIPS did not reveal morphological and chemical changes even after 14-day incubation (data not shown). All composite membranes showed the evolution of the calcium phosphate layer formation process during the in vitro bioactivity test. Just after 3 days of immersion, both surfaces of all composites were rich in calcium (Ca) and phosphorus (P). Furthermore, a small amount or lack of silicon (Si) derived from glass particles was detected, indicating that the layer uniformly covered the surfaces of composite membranes. The layers morphologies and $\mathrm{Ca} / \mathrm{P}$ molar ratios (Fig. 5a) suggested the formation of HAp precursors (ACP, OCP). As the immersion time increased, distinct spherical crystals appeared 
Table 1 Melting temperature $\left(T_{\mathrm{m}}\right)$ and crystallinity $\left(\chi_{\mathrm{c}}\right)$ of polymer matrix of obtained membranes

\begin{tabular}{lll}
\hline Material & $T_{\mathrm{m}}\left({ }^{\circ} \mathrm{C}\right)$ & $\chi_{\mathrm{c}}(\%)$ \\
\hline PCL/TIPS & $59.17 \pm 0.19$ & $60.25 \pm 1.26$ \\
PCL/A2 $<3 \mu \mathrm{m} / \mathrm{TIPS}$ & $54.50 \pm 0.20$ & $30.60 \pm 1.15$ \\
$\mathrm{PCL} / \mathrm{A} 2<45 \mu \mathrm{m} / \mathrm{TIPS}$ & $54.67 \pm 0.14$ & $30.60 \pm 0.99$ \\
$\mathrm{PCL} / \mathrm{NIPS}$ & $53.33 \pm 0.28$ & $29.19 \pm 1.26$ \\
$\mathrm{PCL} / \mathrm{A} 2<3 \mu \mathrm{m} / \mathrm{NIPS}$ & $53.67 \pm 0.11$ & $19.77 \pm 1.53$ \\
PCL/A2 $<45 \mu \mathrm{m} / \mathrm{NIPS}$ & $54.54 \pm 0.32$ & $20.31 \pm 1.44$ \\
\hline
\end{tabular}

Results are expressed as mean $\pm \mathrm{SD}(n=3)$

on the surfaces of the composites and grew in size. Depositions exhibited spherical cauliflower-like morphology, typical of carbonated hydroxyapatite (HCA) [1]. It seemed that faster formation of welldeveloped crystal forms covering entire surfaces of samples after 14-day incubation occurred for membranes containing smaller BG particles $(<3 \mu \mathrm{m})$.

The Ca/P molar ratios of layers formed on the GS surfaces of composite membranes upon immersion in SBF were evaluated with EDX analysis. The results of $\mathrm{Ca} / \mathrm{P}$ molar ratios as a function of immersion time are shown in Fig. 6a. The Ca/P ratios of layers on the composite surfaces gradually increased over time; however, some differences between materials were observed. After 3 days of incubation, layers developed on the surfaces of membranes containing BG particles of $<45 \mu \mathrm{m}$ size were characterized by lower $\mathrm{Ca} / \mathrm{P}$ molar ratios compared to materials with smaller BG particles $(<3 \mu \mathrm{m})$. In particular, layers on the PCL/A2 $<45 \mu \mathrm{m} / \mathrm{NIPS}$ and PCL/A2 $<45 \mu \mathrm{m} /$ TIPS materials exhibited $\mathrm{Ca} / \mathrm{P}$ molar ratios in the range of 1.16-1.21, which can indicate the formation of amorphous calcium phosphate (ACP) [29]. In turn, for the PCL/A2 $<3 \mu \mathrm{m} / \mathrm{NIPS}$ membrane the ratio was 1.33, what is a characteristic value of octacalcium phosphate (OCP) [29], and 1.42 for the PCL/ A2 $<3 \mu \mathrm{m} /$ TIPS composite. After 7 days of incubation, the ratios were similar for all composite membranes and reached values in the range of 1.50-1.57, what is typical for calcium-deficient hydroxyapatite (CDHA, 1.50-1.67). Further incubation of membranes obtained with TIPS method resulted in the increase in $\mathrm{Ca} / \mathrm{P}$ ratios (1.60-1.63 after 14 days); however, the ratios still remained in the range of values characteristic for CDHA. In turn, the layers formed on the $\mathrm{PCL} / \mathrm{A} 2<45 \mu \mathrm{m} / \mathrm{NIPS}$ and PCL/A2 $<3 \mu \mathrm{m} / \mathrm{NIPS}$ membranes showed significant increase in $\mathrm{Ca} / \mathrm{P}$ ratios (1.81 and 1.92, respectively), which was higher than the stoichiometric value for hydroxyapatite (1.67). This can indicate the formation of a carbonated HAp of B-type HCA (HAp with carbonate ions $\mathrm{CO}_{3}{ }^{2-}$ substituting for phosphate ions $\mathrm{PO}_{4}{ }^{3-}$ ) [30].

\section{ATR-FTIR analysis}

Figure 5a-f shows ATR-FTIR spectra of the GS and AS surfaces of obtained membranes before and after 3, 7 and 14-day incubation in SBF. In case of both polymer membranes, no significant changes indicating the formation of CaP layer after 14-day incubation in SBF were found (Fig. 6a, d). FTIR spectra of all composite materials revealed appearance of new two bands in the range of $560-602 \mathrm{~cm}^{-1}$ and single intensive band at $1020 \mathrm{~cm}^{-1}$ originated from CaP precipitations just after 3 days of soaking, and gradual reduction in intensity of bands characteristic for PCL. New bands can be assigned to O-P-O bending mode and $\mathrm{P}-\mathrm{O}$ stretching mode, respectively [31]. The reduction in intensity of PCL bands can be connected with increasing thickness and/or tightness of $\mathrm{CaP}$ layer covering material surface. Therefore, by comparing ATR-FTIR spectra of materials after the lengthening periods of incubation in SBF, the layer formation kinetics can be estimated. For the GS and AS surfaces of membranes with smaller BG particle size (PCL/A2 $<3 \mu \mathrm{m} / \mathrm{TIPS}$ and PCL/A2 $<3 \mu \mathrm{m} /$ NIPS), the bands originating from PCL vanished at a similar rate, which can suggest similar rate of $\mathrm{CaP}$ layer development on both surfaces. The situation was different with regard to membranes with BG particles of $<45 \mu \mathrm{m}$ size. In case of GS surface, especially of the PCL/A2 $<45 \mu \mathrm{m} / \mathrm{TIPS}$ membrane, significantly faster decrease in intensities of bands arising from PCL compared to AS surface was observed. The rate of band intensity reduction for GS surfaces of membranes with BG particles of $<45 \mu \mathrm{m}$ size was similar to that rate for both surfaces of membranes with smaller BG particles $(<3 \mu \mathrm{m})$. Moreover, it seems that the development of a $\mathrm{CaP}$ layer occurred faster for AS surface of membrane obtained with the NIPS method (PCL/A2 $<45 \mu \mathrm{m}$ / NIPS) compared to the same surface of the PCL/ A2 $<45 \mu \mathrm{m} /$ TIPS material.

\section{ICP-OES}

The changes in the concentrations of $\mathrm{Ca}, \mathrm{P}$ and $\mathrm{Si}$ in the SBF during membrane incubation are shown in 


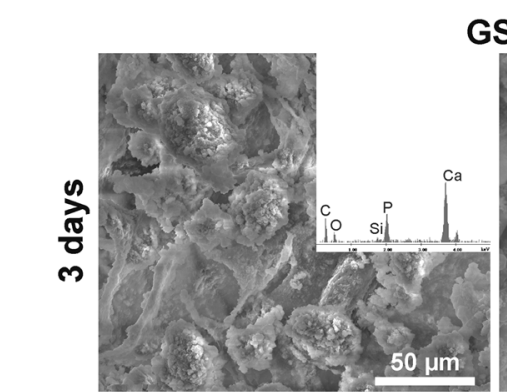

GS
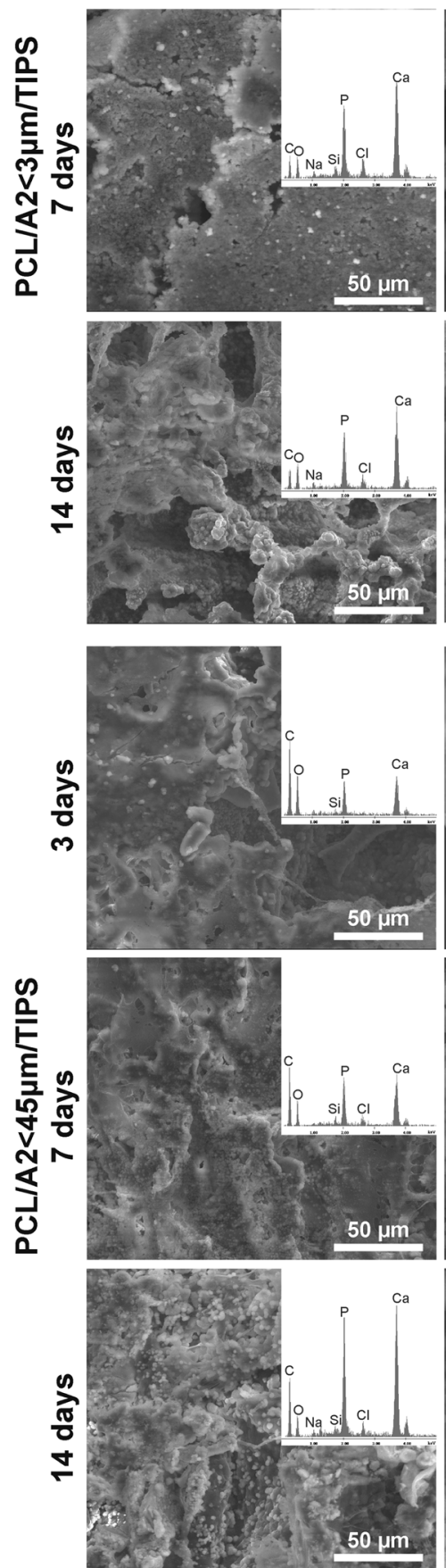
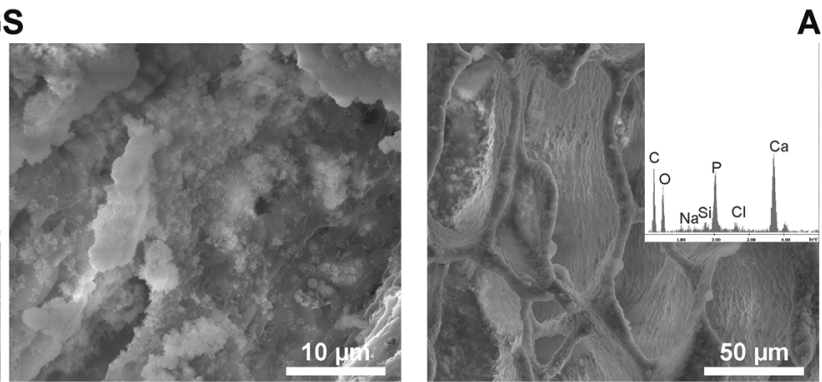

AS
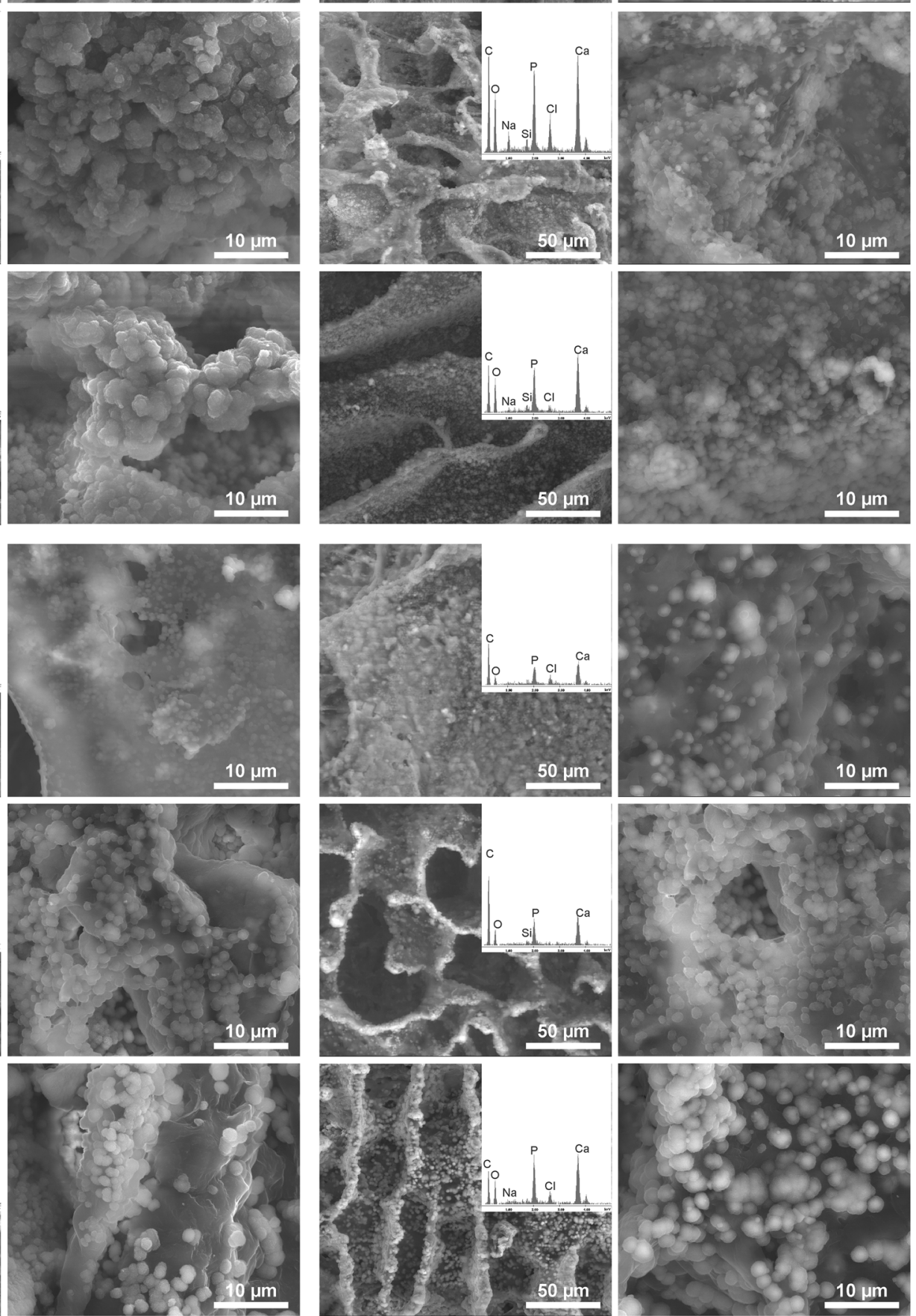

Figure 3 SEM images and EDX spectra (averaged for the entire analysed surface) of both GS and AS surfaces of composite membranes obtained with the use of TIPS method after 3-, 7- and 14-day incubation in SBF. 


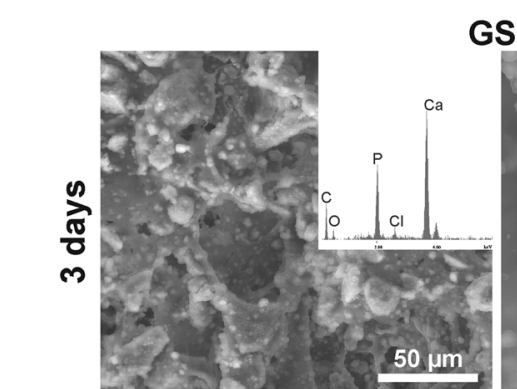

GS
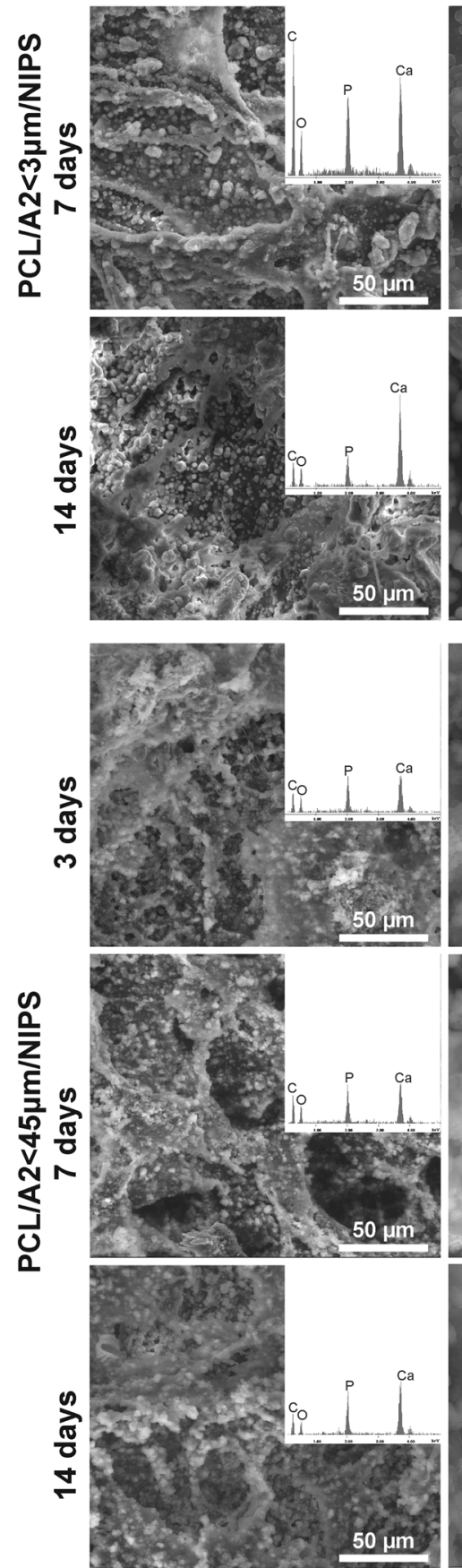
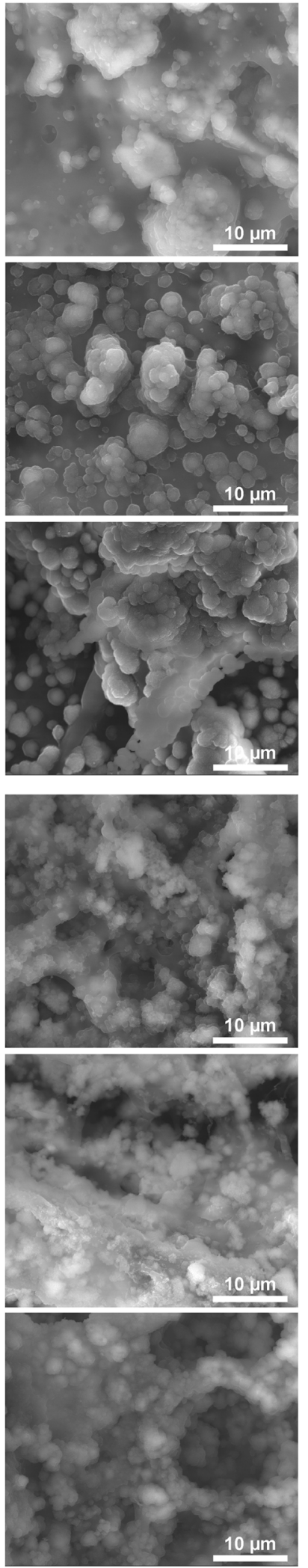

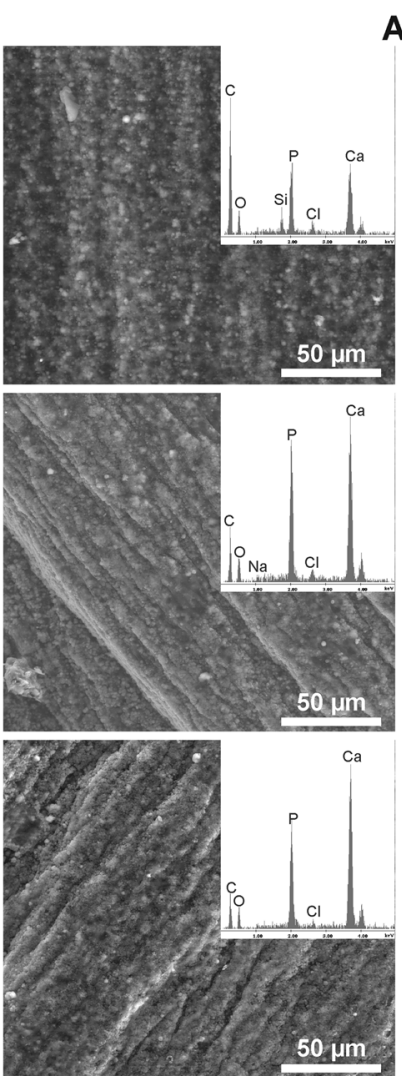

AS
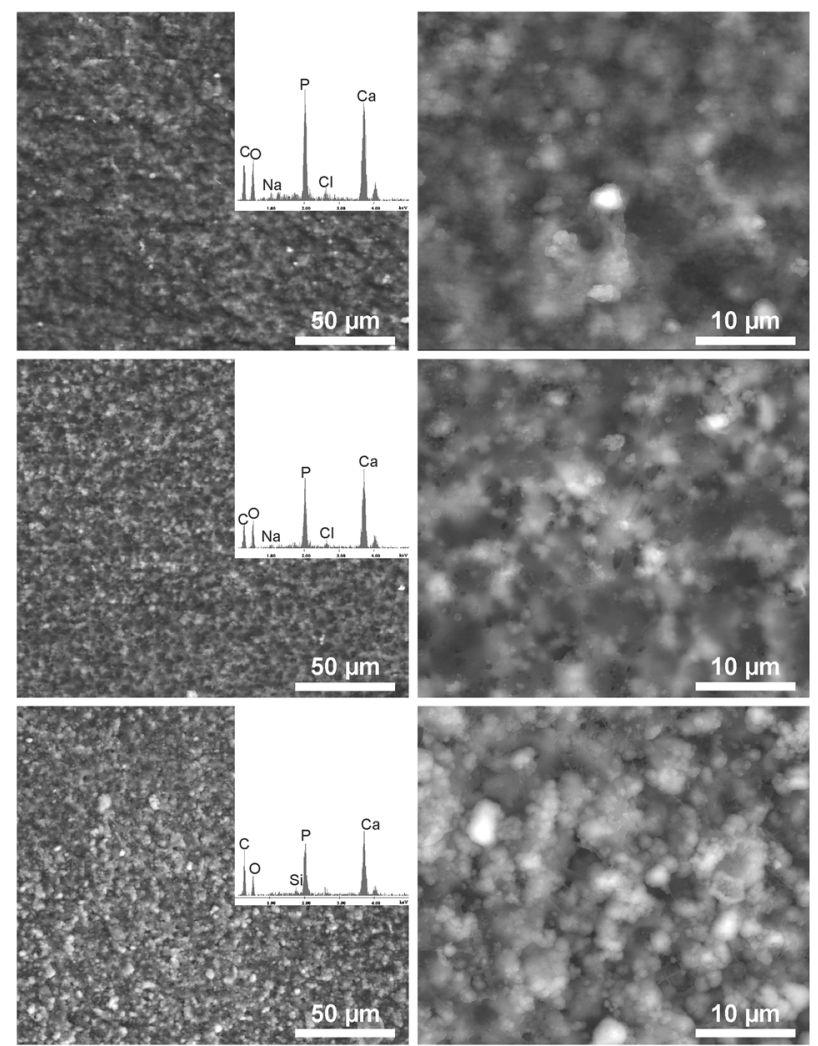

Figure 4 SEM images and EDX spectra (averaged for the entire analysed surface) of both GS and AS surfaces of composite membranes obtained with the use of NIPS method after 3-, 7- and 14-day incubation in SBF. 
(a)
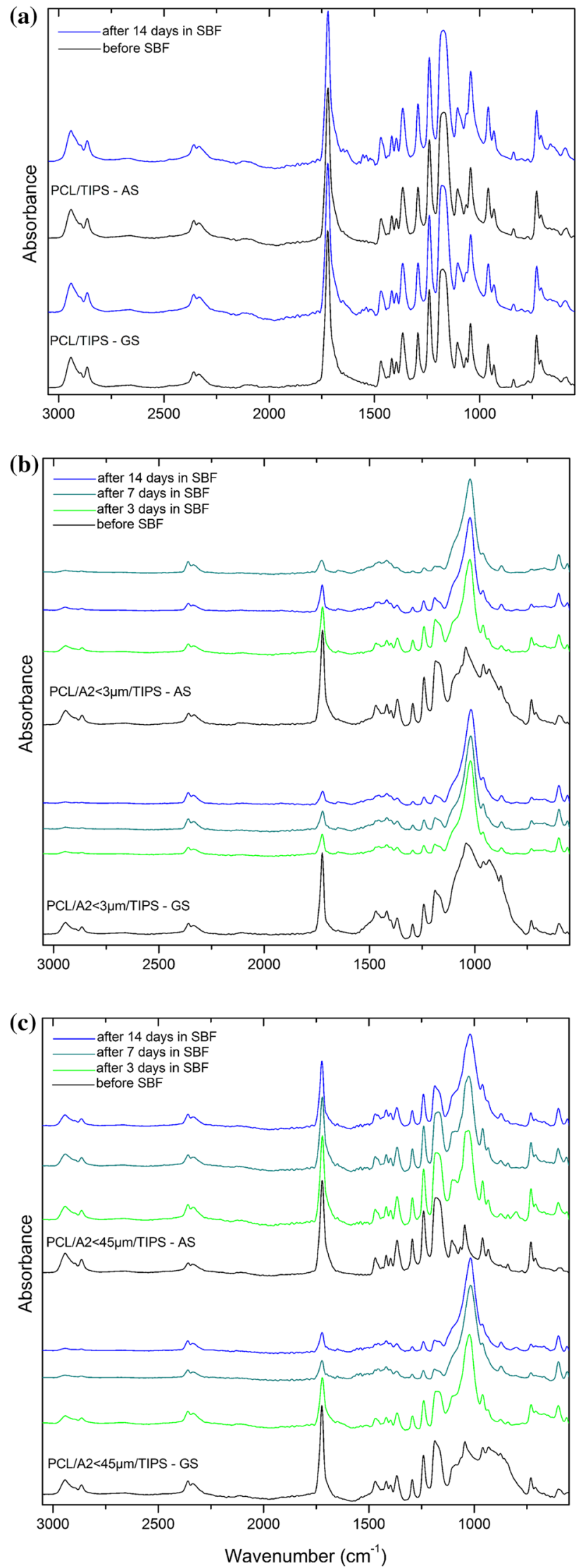
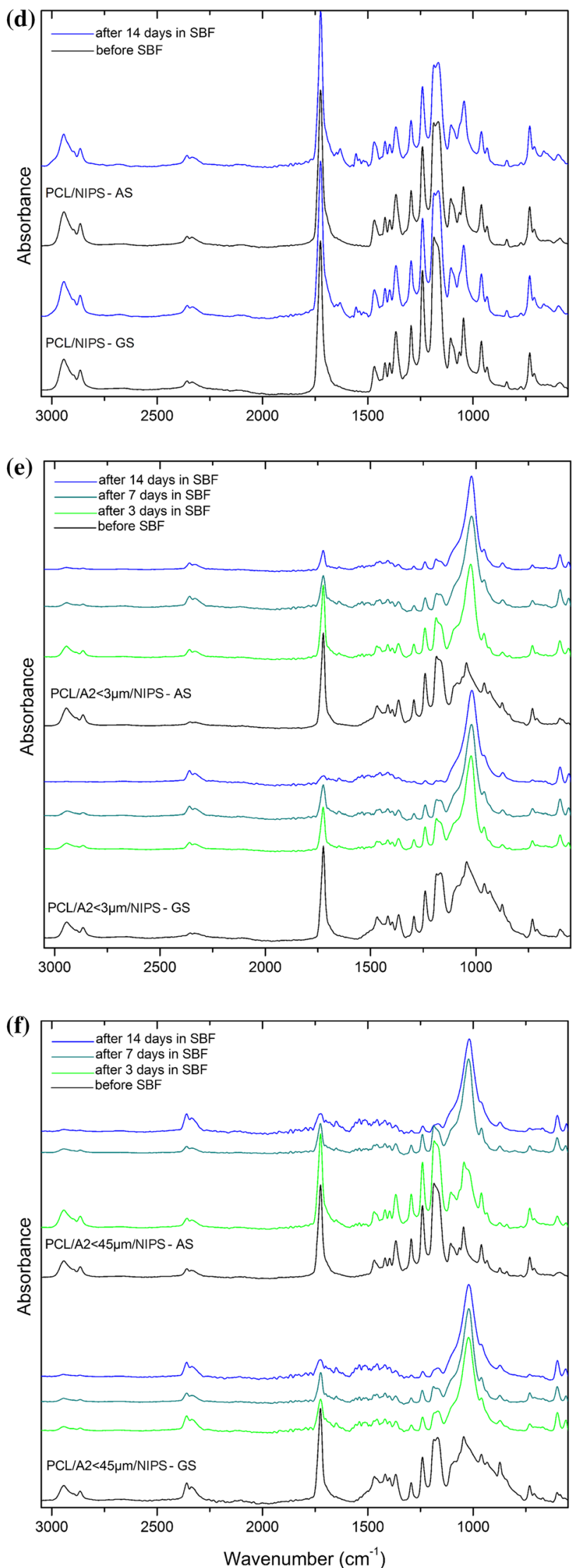
4 Figure 5 ATR-FTIR spectra of both GS and AS surfaces of PCL/ TIPS (a), PCL/A2 $<3 \mu \mathrm{m} /$ TIPS (b), PCL/A2 $<45 \mu \mathrm{m} / \mathrm{TIPS}$ (c), PCL/NIPS (d), PCL/A2 $<3 \mu \mathrm{m} / \mathrm{NIPS}$ (e), PCL/A2 $<45 \mu \mathrm{m} / \mathrm{NIPS}$ (f) membranes before and after 3-, 7- and 14-day incubation in SBF.

Fig. $6 \mathrm{~b}-\mathrm{d}$, respectively. Ion concentrations in SBF for both polymer PCL/TIPS and PCL/NIPS membranes did not change significantly over soaking time. In general, Ca concentration in SBF was affected both by the release from the glass structure and the consumption of Ca resulting from formation of calcium phosphate layer on the material surfaces during incubation [32]. The variations in the Ca concentrations for the composite materials were dependent on preparation method (Fig. 5b). Furthermore, the Ca release/consumption profiles for the composites
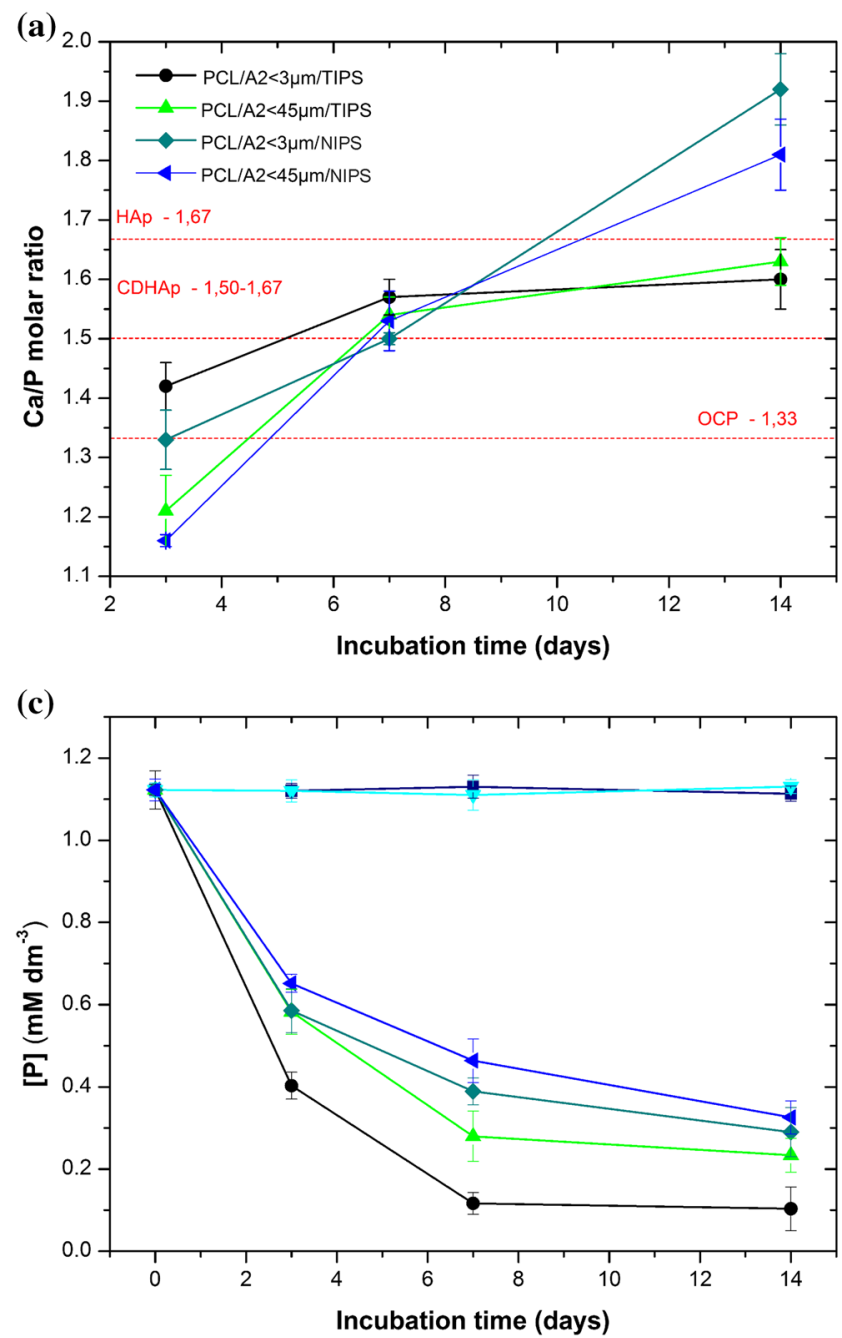

containing $\mathrm{A} 2<45 \mu \mathrm{m}$ and $\mathrm{A} 2<3 \mu \mathrm{m}$ glass particles showed similar trend within TIPS and NIPS groups. In case of the PCL/A2/TIPS membranes, the $\mathrm{Ca}$ concentrations did not change significantly up to 3 days of incubation, after which the Ca content in SBF gradually decreased. The highest depletion of Ca in SBF was observed from day 7 to day 14; however, the decrease was greater for the membrane with larger BG particle size (PCL/A2 $<45 \mu \mathrm{m} / \mathrm{TIPS}$ ). In contrary, for the PCL/A2/NIPS membranes Ca concentrations started to decrease just in the first 3 days of incubation. Moreover, consumption of $\mathrm{Ca}$ at the first stage of soaking was the fastest. Then, Ca content in SBF gradually decreased from day 3 to day 14 and at the end of incubation ion concentration reached lower value for the membrane with smaller BG particle size (PCL/A2 $<3 \mu \mathrm{m} / \mathrm{NIPS}$ ). These differences
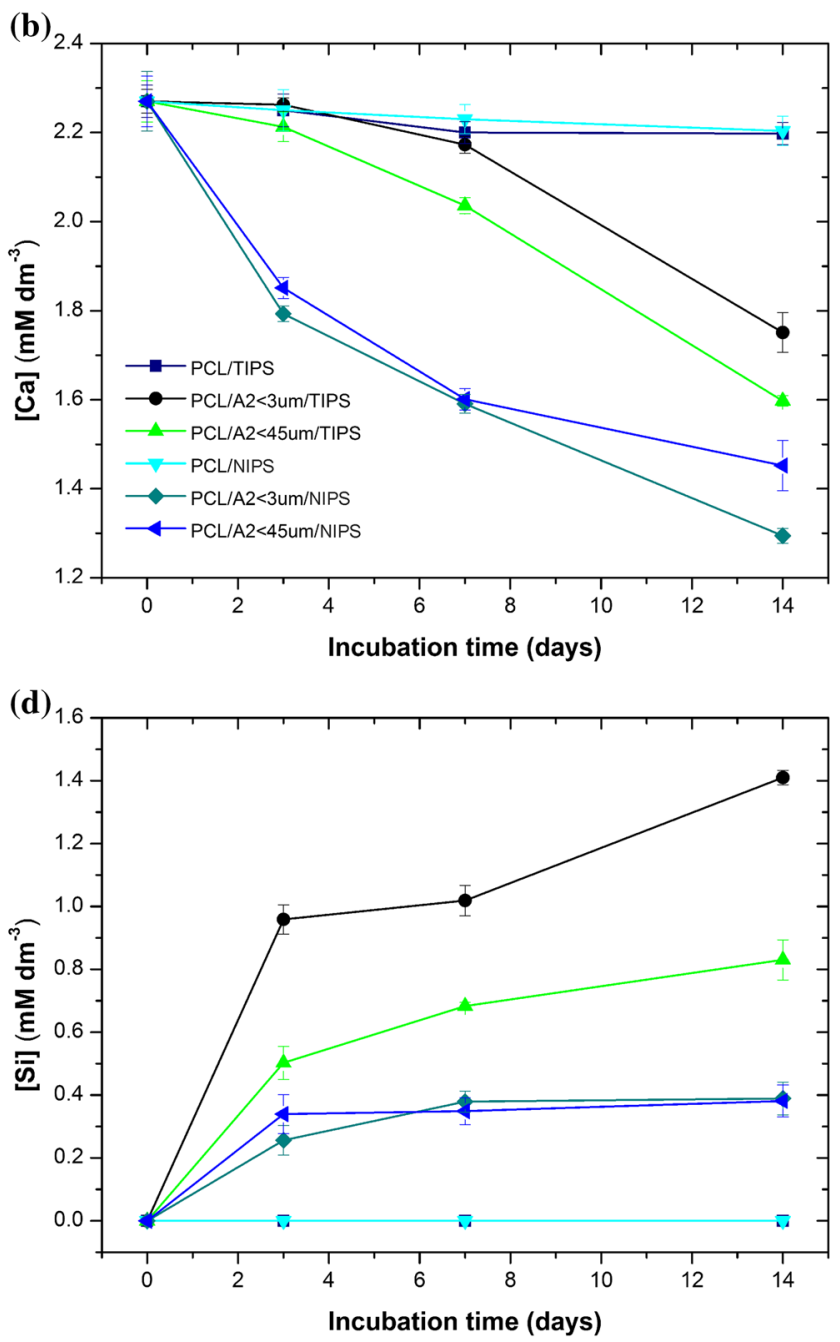

Figure 6 Variations of the $\mathrm{Ca} / \mathrm{P}$ molar ratio of the formed layer after incubation in $\mathrm{SBF}(\mathbf{a})$ and the changes of $\mathrm{Ca}(\mathbf{b})$, $\mathrm{P}(\mathbf{c})$ and $\mathrm{Si}$ (d) concentrations in SBF during 14-day soaking. 
between TIPS and NIPS groups probably resulted from variations in $\mathrm{Ca}$ release profiles. It can be observed that the release rate of $\mathrm{Si}$ to SBF from membranes obtained with the use of TIPS method was significantly higher (especially for the PCL/ A2 $<3 \mu \mathrm{m} /$ TIPS membrane) compared to materials produced with NIPS method (Fig. 6d). This can suggest that the release rate of Ca was also higher for these materials and consequently it compensated the changes in ion concentrations resulting from their uptake caused by formation of $\mathrm{CaP}$ layer. In addition, this interpretation can be supported by the fact that after 3 days of incubation, when $\mathrm{CaP}$ layer was developed and simultaneously further $\mathrm{Si}$ and $\mathrm{Ca}$ release from material structure was reduced, $\mathrm{Ca}$ depletion was accelerated.

These findings coincided with the decrease in $\mathrm{P}$ concentration confirming $\mathrm{CaP}$ layer formation on the surfaces of composite membranes. The $\mathrm{P}$ consumption profiles for each of the composite membranes showed similar trend (Fig. 6c). In the first 3 days of incubation, the decrease in $\mathrm{P}$ content was the fastest. It can be observed that the reduction in ion content in SBF for composites obtained with TIPS method was higher (especially for the PCL/A2 $<3 \mu \mathrm{m} / \mathrm{TIPS}$ membrane) compared to membranes obtained with the use of NIPS method.

\section{In vitro osteoblast response}

As shown in Fig. 7a, a significant increase in the number of intact adherent cell between 7 and 14 days of culture on all tested membranes was observed. Nevertheless, the values recorded for the composite membranes prepared with the use of TIPS method (PCL/A2 $<3 \mu \mathrm{m} /$ TIPS and PCL/A2 $<45 \mu \mathrm{m} / \mathrm{TIPS}$ ) after both culture periods were significantly lower compared to other membranes and control material (TCPS). Moreover, for these latter membranes and TCPS, after 14 days of culture, cell number was on similar level. No significant effect of BG particle size on cell proliferation was detected.

Osteoblasts cultured for 7 days on all membranes were uniformly distributed as it was shown in Fig. 7e for the PCL/A2 $<3 \mu \mathrm{m} / \mathrm{TIPS}$. They were able to penetrate into the pores and adhere to the pore surfaces, creating a three-dimensional cell-cell network.

Cytotoxicity of membranes, estimated based on the measurement of adenylate kinase (AK) released from the damaged cells, is shown in Fig. $7 \mathrm{~b}$. After 7 and 14 days of culture, cytotoxicity of all obtained materials was similar or even lower compared to TCPS, indicating no cytotoxic effects on osteoblasts. Moreover, cytotoxicity of all membranes decreased over time.

ALP activity was measured after 7 days of culture, as an early marker of osteoblast differentiation. The results were presented as ALP activity normalized to cell number (Fig. 7c). The results showed that osteoblasts cultured on composite membranes obtained with both TIPS and NIPS methods showed significantly higher ALP activity compared to cells in contact with the polymer PCL/TIPS and PCL/NIPS membranes, as well as the TCPS, for which the activity was on similar level. What is more, the ALP activity of cells both on the PCL/A2 $<3 \mu \mathrm{m} / \mathrm{TIPS}$ and PCL/A2 $<45 \mu \mathrm{m} /$ TIPS membranes was the highest. This early differentiation characteristic can be correlated with the decrease in proliferation rate after 7 and 14 days of culture [33]. No significant effect of BG particle size on ALP activity of cells in contact with materials was observed.

For all membranes and control material (TCPS), matrix mineralization between 14 and 21 days of culture was enhanced; however, the increase was significantly higher for composite materials (Fig. 7d). After 14 and 21 days of culture, osteoblasts cultured on composites obtained with both TIPS and NIPS methods showed significantly higher level of mineralization compared to cells on the polymer PCL/TIPS and PCL/NIPS membranes, as well as on the TCPS. As in case of ALP activity, osteoblasts cultured for 21 days on composites prepared with the use of TIPS method exhibited the highest matrix mineralization. After both culture periods, between the PCL/ A $2<3 \mu \mathrm{m} / \mathrm{NIPS}$ and the PCL/A2 $<45 \mu \mathrm{m} / \mathrm{NIPS}$ membranes there were no statistically significant differences. In contrary, within the group of materials prepared with the use of TIPS method, cells cultured for 14 and 21 days on membrane with BG particles of $<3 \mu \mathrm{m}$ size showed significantly higher values. Matrix mineralization process was also confirmed with SEM/EDX analysis (Fig. 7f). After 21 days of culture, a confluent layer of osteoblasts (confirmed by the presence of sulphur in EDX analysis) covered with mineral deposits rich in calcium and phosphorus was observed on the surface of the PCL/ A2 $<3 \mu \mathrm{m} /$ NIPS membrane. 
(a)

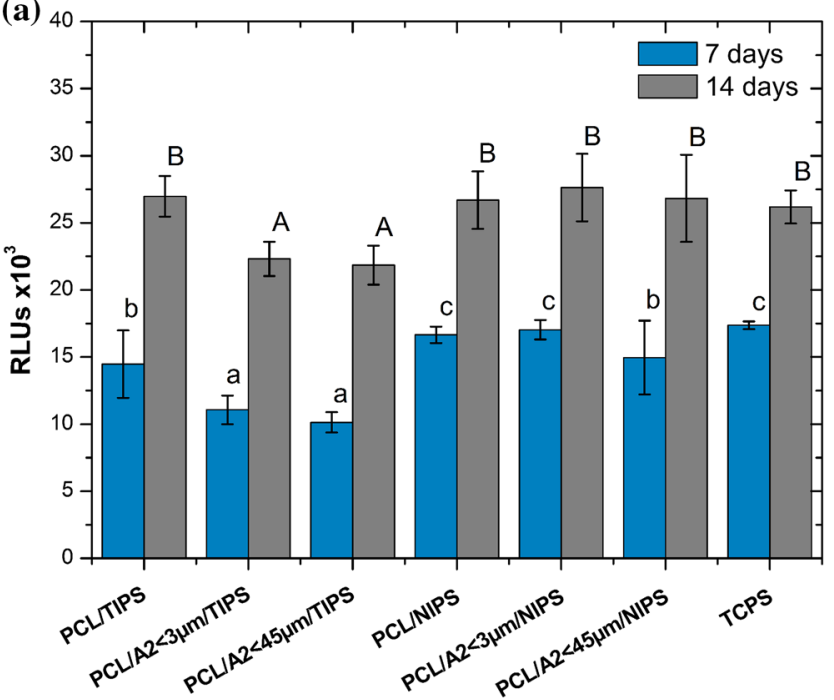

(c)

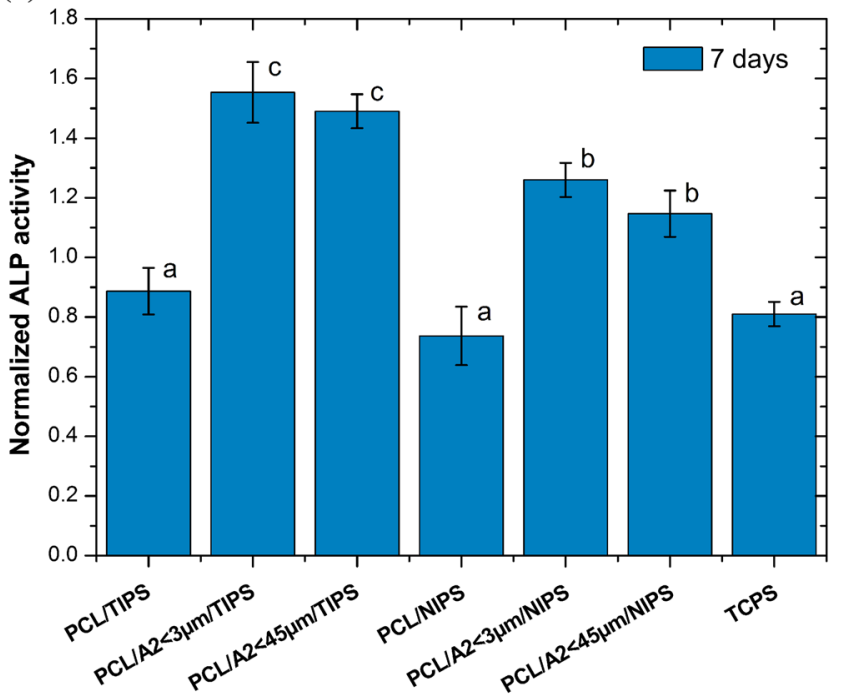

(b)

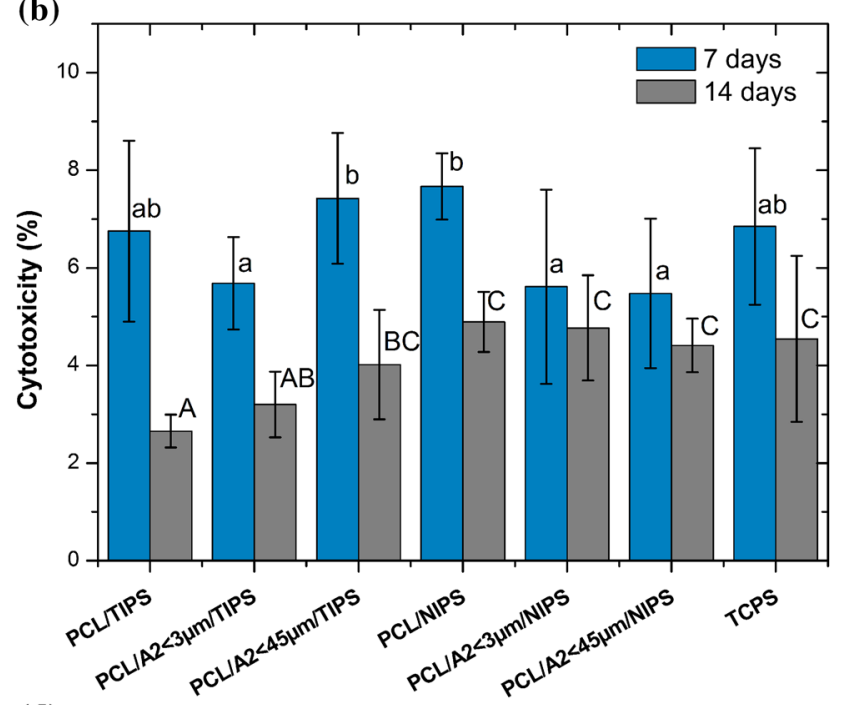

(d)

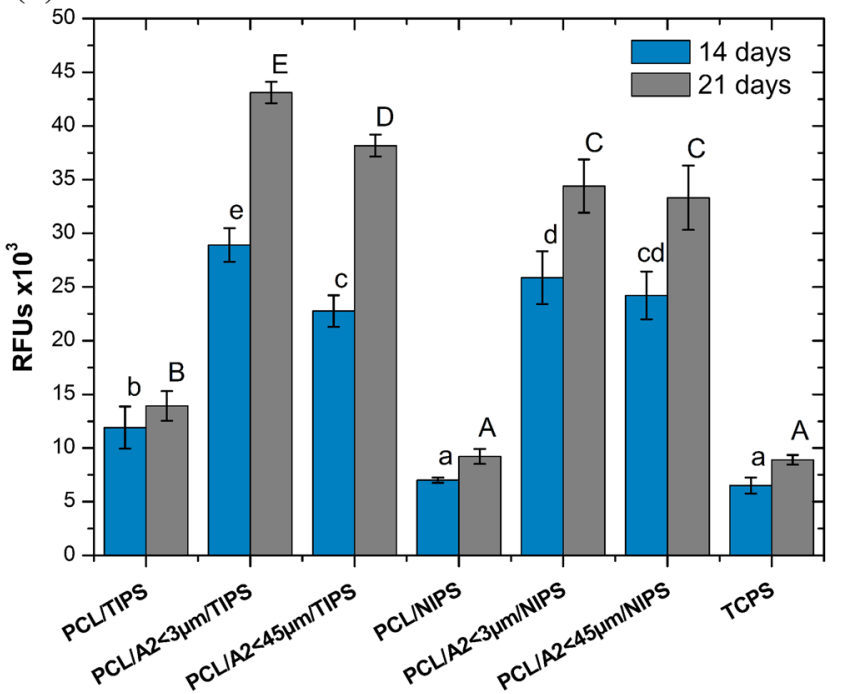

(e)

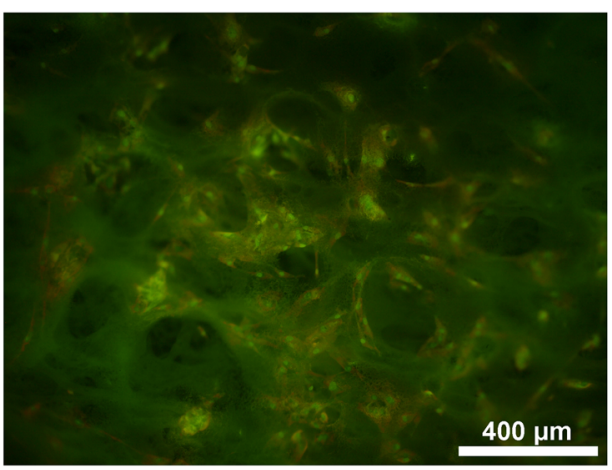

(f)

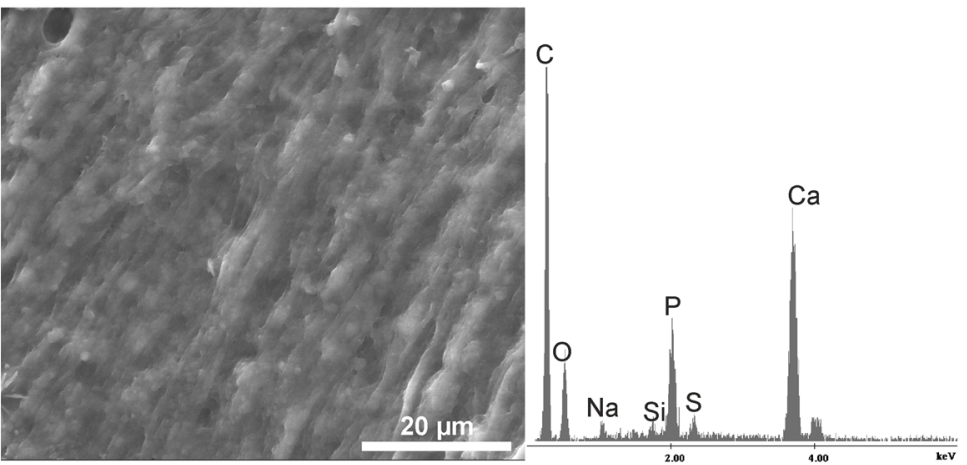

Figure 7 Adenylate kinase (AK) level in the lysate corresponding to the number of intact adherent cells (a), AK level in the supernatant related to AK level in the lysate representing material cytotoxicity (b), ALP activity normalized to cell number (c), ECM mineralization (d). Results are expressed as mean \pm SD. Statistically significant differences $(p<0.05)$ between each membrane and TCPS after different cell culture periods are indicated by upper and lower case, respectively. Fluorescence microscope image of osteoblasts grown on the PCL/A2 $<3 \mu \mathrm{m} / \mathrm{TIPS}$ membrane for 7 days (e), SEM image and EDX spectrum of a osteoblast layer on the PCL/A2 $<3 \mu \mathrm{m} / \mathrm{NIPS}$ membrane after 21 days of culture (f). 


\section{Discussion}

Membranes for tissue engineering should possess adequate porosity, pore size and shape, suitable surface properties, including wettability, depending on specific applications. What is more, materials for bone regeneration should exhibit excellent and controllable bone-bonding ability, support osteoblast proliferation, induce their differentiation and extracellular matrix mineralization. In present work, we have proved that not only material properties, but also biological features of the membranes can be modified using proposed preparation methods in combination with different glass particle sizes.

The differences between AS and GS surfaces morphologies of the PCL/TIPS material probably arose from various cooling rates of both surfaces. This might have resulted in the different solvent crystallization kinetics (nucleation and growth of 1,4-dioxane crystals in the polymer solution) and therefore the different pore architectures [34]. The introduction of BG particles into the polymer solution can hinder the solvent crystallization process to some extent and thereby reduce porosity of GS surface of the PCL/A2/TIPS membranes due to intensive sedimentation of $<45 \mu \mathrm{m}$ BG particles [12]. Obtained morphologies of NIPS membranes resulted from good solvent-non-solvent (DMF-water) miscibility. The higher the miscibility between the solvent and non-solvent is, the more likely instantaneous demixing will occur and more porous membrane will be obtained. In case of low miscibility, an asymmetric membrane with a dense nonporous top layer is likely to be obtained [35].

According to Tang et al. [36], PCL molecules can aggregate in different ways, depending on the polarity of the solvent. For solvent with higher polarity, polar "hydro-neutral" ester groups -C(O)O- [37] in PCL tend to be presented on the surface, while non-polar, highly hydrophobic polymer backbone chain tends to aggregate away from the polar solvent. Therefore, the surface of a material tends to be relatively more hydrophobic when solvent with lower polarity is used. It seems that this correlation is consistent with the results obtained in present work. The PCL/TIPS membrane, obtained from solvent with lower polarity (1,4-dioxane, polarity 16.4 [38]), showed higher contact angle values (AS- $121.57^{\circ}$; GS-107.45 ) compared to the PCL/NIPS material (AS-70.63; GS-85.36 ${ }^{\circ}$ ) produced using $\mathrm{N}, \mathrm{N}$-dimethylformamide (polarity 40.4). What is more, during manufacturing process of the PCL/NIPS membrane, direct exposure of the polymer solution to a highly polar liquid (water) could have led to similar polymer chain behaviour as in polar solvent.

The differences in water contact angle values between individual membranes and also between their two tested surfaces can result from various surface topography, surface porosity and pore size, as well as surface composition, namely the presence and distribution of glass particles in the polymer matrix. It is known that for hydrophobic materials (i. a. PCL) surface roughness enhances the hydrophobicity $[39,40]$. On the other hand, the water droplet can be spread easier on the more porous surface of membrane, which results in higher hydrophilicity [41]. It was previously shown that bioactive glass particles, as a hydrophilic material, remarkably improve the hydrophilicity of PCL matrix [9, 18]. Nevertheless, a more pronounced effect was observed for composites containing smaller bioactive glass particles due to their homogenous distribution in the PCL matrix and thus uniform exposition on the surfaces [42, 43].

Polymer crystallinity is one of the factors that affect many of the material properties such as mechanical behaviour [44, 45], degradation rate [20, 46], bioactivity [18], as well as cellular response [47, 48]. The differences in crystallinity and melting temperature may be due to various rates of membrane formation and different phase separation mechanisms in the NIPS and TIPS techniques. In case of NIPS method, fast polymer solidification rate was induced by immediate exchange of solvent and non-solvent after immersion in coagulation bath, and therefore polymer chain migration was blocked. Good solvent-nonsolvent (DMF-water) miscibility promoted fast solvent-non-solvent exchange, and thus fast membrane formation [35]. In TIPS technique, when a polymer solution is cooled, the nucleation/crystallization of the solvent gradually occurs. The remaining polymer solution starts to demix because of the loss of the solvent in the liquid phase and the reduced solubility of polymer at lower temperatures. Demixing/phase separation process leads to increase in the concentration of polymer in the remaining liquid phase. Polymer chains have more time to crystallize, what may promote the crystallization process to yield higher crystallinity and melting temperature [49].

The presence of bioactive glass particles in polymer matrix reduced their melting temperature and degree 
of crystallinity. Such behaviour suggests the hindering effect of glass particles on crystallization process caused by the limitation of PCL chain mobility and therefore the reduction in ability to rearrange into ordered regions [20]. In case of the materials obtained with TIPS method, significant reduction in melting temperature and degree of crystallinity (by half) was observed. On the other hand, glass particles of both sizes practically did not change $T_{\mathrm{m}}$ of the PCL/A2/ NIPS membranes, while $\chi_{c}$ values were reduced (about 30\%), but not to the same extent as in case of the PCL/A2/TIPS composites. That also confirms that thermal-induced phase separation occurred slower than non-solvent-induced process, providing more time for polymer crystallization. The changes of $T_{\mathrm{m}}$ and $\chi_{\mathrm{c}}$ seemed to be irrespective of glass particle size.

The results of in vitro bioactivity test can suggest that $\mathrm{CaP}$ layer formation on the surfaces of obtained membranes occurred through ACP-OCP-CDHAHCA transformation process, however, in a different rate depending on the $\mathrm{BG}$ particle size and membrane preparation method. In the literature, process of the CDHA layer formation on the conventional sol-gel glass surface upon immersion in SBF occurs through the direct crystallization of ACP [50]. Template (mesoporous) glasses show the same trend concerning the initial formation of an ACP layer upon soaking in SBF; however, additional intermediate phase OCP formation and its maturation into CDHA take place [29]. ACP-OCP-CDHA maturation process was reported as similar to in vivo bone biomineralization $[42,44]$. In case of bioactive glasses, bioactivity process depends mainly on their chemical composition, as well as textural (porosity, specific surface area) and structural characteristics $[21,22,50]$. Nevertheless, when considering bioactive response of composite materials, in which bioactive glass particles are dispersed in polymer matrix, other parameters such as BG particle distribution in matrix, composite porosity, specific surface area, surface topography and wettability must also be taken into account.

According to Tamjid et al. [18] submicron BG particles improve the nanotopography and hydrophilicity of the PCL film surface and consequently enhance composite bioactivity in contrast to BG microparticles. Furthermore, the larger surface area of BG particles allows a faster ion exchange with the surrounding medium enhancing ability of HAp layer formation on the composite surfaces [31]. Our previous studies indicated that the uniform distribution of smaller BG particles $(\mathrm{A} 2<3 \mu \mathrm{m})$ in the PCL matrix of solvent-cast films resulted in crystallization of carbonated hydroxyapatite (HCA) on the AS surface. In contrary, the same surface of films with A2 glass particles of $<45 \mu \mathrm{m}$ size revealed no significant morphological and chemical changes after incubation in SBF [42]. In present work, CaP layer formation occurred on both surfaces of membranes containing smaller particles size (PCL/A2 $<3 \mu \mathrm{m} / \mathrm{TIPS}$ and $\mathrm{PCL} / \mathrm{A} 2<3 \mu \mathrm{m} / \mathrm{NIPS})$ at similar rate. Nevertheless, AS surfaces of both materials containing larger-sized particles $(<45 \mu \mathrm{m})$ were also covered with bioactive layer just after 3 days of incubation. This may result from high porosity of obtained membranes in contrast to above-mentioned solvent-cast films. Moreover, higher rate of layer development on AS surface of the PCL/A2 $<45 \mu \mathrm{m} / \mathrm{NIPS}$ membrane compared to the same surface of the PCL/A2 $<45 \mu \mathrm{m} / \mathrm{TIPS}$ may occur because of improved wettability and also differences in topographic/microstructural characteristics of these two membranes.

Jaakkola et al. [51] and Caridade et al. [31] observed that faster consumption of both $\mathrm{Ca}$ and $\mathrm{P}$, as well as faster release of $\mathrm{Si}$ occurred in case of composites containing smaller BG particles size. Apart from Ca release/consumption profiles affected by above-mentioned compensation process, these findings correlate with results obtained in present work only for materials produced with the use of TIPS method. In case of composites obtained with NIPS technique, release/consumption profiles practically did not depend on particle size of BG. Probable reason for this differences and also variations in $\mathrm{Si} /$ Ca release between materials fabricated with the use two methods result from differences in distribution of BG particles in the polymer matrix. In our previous work [10] the effect of preparation method of PCL/ BG composite scaffolds on their bioactivity was also observed.

The results indicate that PCL/BG composite membranes can stimulate osteoblast differentiation and also matrix mineralization process. Similar effect of the BG particles addition into polymer matrix on osteoblastic cell activity was reported in the literature $[52,53]$. Furthermore, it seems that osteoblast response was dependent mainly on preparation method of materials. This may be due to differences in $\mathrm{Ca}$ and $\mathrm{Si}$ release from membranes prepared with 
TIPS and NIPS methods, as shown in the ICP-OES analysis (Fig. 6b, d). The soluble $\mathrm{Ca}$ and $\mathrm{Si}$ have been shown to stimulate the genes that control osteoblast differentiation in vitro [54], as well as promote their proliferation and collagen secretion [55] when present at a particular ratio and at a particular concentration range in the culture medium. On the other hand, diversified microstructure of the membranes prepared with two different techniques may be another explanation for the various osteoblast behaviours. Osteogenic differentiation in vitro has been shown to depend on pore size [56] and material porosity [57]. Our previous work has indicated that preparation methods of PCL/BG composite scaffolds affected ALP activity of normal human osteoblasts [10].

\section{Conclusions}

In present work, the role of membrane preparation methods (TIPS and NIPS) and their modifications with gel-derived bioactive glass particles of two different sizes $(<45$ and $<3 \mu \mathrm{m})$ in modulating membrane microstructure, surface wettability, polymer matrix crystallinity and kinetics of in vitro bioactivity, as well as osteoblast response was investigated. Our results indicated a possibility to control microstructure (pore size ranging from submicron to hundreds of micrometres), wettability (from hydrophobic to fully wettable surface) and polymer crystallinity (from 19 to 60\%) in a wide range by the use of various preparation methods and different BG particle sizes.

Obtained composite membranes showed excellent in vitro bioactivity, as evidenced by the formation of uniform calcium phosphate layer on their surfaces just after 3-day immersion in SBF. Moreover, we have demonstrated that bioactive layer formation on the surfaces of membranes occurred through ACP-OCPCDHA-HCA transformation, that mimic in vivo bone biomineralization process. The presence of BG particles was shown to impart osteoinductive properties to obtained membranes, supporting normal human osteoblast attachment and proliferation, as well as stimulating cell differentiation and also matrix mineralization process in vitro. This proves that obtained membranes can actively promote regeneration of bone tissue. We showed that kinetics of bioactivity process and also osteoinductive properties of membranes can be easily modulated with the use of proposed variables. We have presented new opportunities to design and fabricate multifunctional composite membranes with tunable physicochemical and biological properties that can meet complex requirements of bone tissue engineering. However, further in vitro and in vivo studies are needed to determine the effect of the preparation methods and BG particle sizes on the biological properties of the composite membranes and to correlate them with their physicochemical parameters and to show real usefulness in BTE.

\section{Acknowledgements}

This work was supported by the National Science Centre, Poland Grant No. 2015/17/N/ST8/00226 (MD). Michal Dziadek acknowledges financial support from the National Science Centre, Poland under doctoral scholarship (2017/24/T/ST8/00041).

\section{Compliance with ethical standards}

Conflict of interest The authors declare that they have no conflict of interest.

Open Access This article is distributed under the terms of the Creative Commons Attribution 4.0 International License (http://creativecommons.org/licen ses/by/4.0/), which permits unrestricted use, distribution, and reproduction in any medium, provided you give appropriate credit to the original author(s) and the source, provide a link to the Creative Commons license, and indicate if changes were made.

\section{References}

[1] Li W, Nooeaid P, Roether JA et al (2014) Preparation and characterization of vancomycin releasing PHBV coated 45S5 Bioglass ${ }^{\circledR}$-based glass-ceramic scaffolds for bone tissue engineering. J Eur Ceram Soc 34:505-514. doi:10.1016/j. jeurceramsoc.2013.08.032

[2] Stodolak-zych E, Frączek-szczypta A, Wiecheć A, Błażewicz M (2012) Nanocomposite polymer scaffolds for bone tissue regeneration. Acta Phys Pol, A 121:518-521

[3] Stodolak-Zych E (2013) Osteoconductive nanocomposite materials for bone regeneration. Mater Sci 730:38-43. doi:10.4028/www.scientific.net/MSF.730-732.38

[4] Bottino MC, Thomas V, Schmidt G et al (2012) Recent advances in the development of GTR/GBR membranes for 
periodontal regeneration - $\mathrm{a}$ materials perspective. Dent Mater 28:703-721. doi:10.1016/j.dental.2012.04.022

[5] Zhang K, Ma Y, Francis LF (2002) Porous polymer/bioactive glass composites for soft-to-hard tissue interfaces. J Biomed Mater Res 61:551-563. doi:10.1002/jbm.10227

[6] Tu C, Cai Q, Yang J et al (2003) The fabrication and characterization of poly(lactic acid) scaffolds for tissue engineering by improved solid-liquid phase separation. Polym Adv Technol 14:565-573. doi:10.1002/pat.370

[7] Suntornnond R, An J, Yeong WY, Chua CK (2015) Biodegradable polymeric films and membranes processing and forming for tissue engineering. Macromol Mater Eng 300:858-877. doi:10.1002/mame.201500028

[8] Dziadek M, Stodolak-Zych E, Cholewa-Kowalska K (2017) Biodegradable ceramic-polymer composites for biomedical applications: a review. Mater Sci Eng, C 71:1175-1191. doi:10.1016/j.msec.2016.10.014

[9] Lei B, Shin K-H, Noh D-Y et al (2013) Sol-gel derived nanoscale bioactive glass (NBG) particles reinforced poly (є-caprolactone) composites for bone tissue engineering. Mater Sci Eng, C 33:1102-1108. doi:10.1016/j.msec.2012. 11.039

[10] Dziadek M, Pawlik J, Menaszek E et al (2015) Effect of the preparation methods on architecture, crystallinity, hydrolytic degradation, bioactivity, and biocompatibility of PCL/bioglass composite scaffolds. J Biomed Mater Res Part B Appl Biomater 103:1580-1593. doi:10.1002/jbm.b.33350

[11] Sawalha H, Schroën K, Boom R (2007) Polylactide films formed by immersion precipitation: effects of additives, nonsolvent, and temperature. J Appl Polym Sci 104:959971. doi:10.1002/app.25808

[12] Wei G, Ma PX (2004) Structure and properties of nanohydroxyapatite/polymer composite scaffolds for bone tissue engineering. Biomaterials 25:4749-4757. doi:10.1016/j.bio materials.2003.12.005

[13] Ma PX, Zhang R, Xiao G, Franceschi R (2001) Engineering new bone tissue in vitro on highly porous poly(?-hydroxyl acids)/hydroxyapatite composite scaffolds. J Biomed Mater Res 54:284-293. doi:10.1002/1097-4636(200102)54: $2<284$ :AID-JBM16>3.0.CO;2-W

[14] Hou Q, Grijpma DW, Feijen J (2003) Preparation of interconnected highly porous polymeric structures by a replication and freeze-drying process. J Biomed Mater Res 67B:732-740. doi:10.1002/jbm.b.10066

[15] Lebourg M, Antón JS, Ribelles JLG (2008) Porous membranes of PLLA-PCL blend for tissue engineering applications. Eur Polym J 44:2207-2218. doi:10.1016/j.eurpolymj. 2008.04.033

[16] Jiang B, Wang B, Zhang L et al (2016) Preparation of poly (L-lactic acid) membrane from solvent mixture via immersion precipitation. Sep Sci Technol 51:2940-2947. doi:10.1080/01496395.2016.1239638

[17] Akbarzadeh R, Yousefi A-M (2014) Effects of processing parameters in thermally induced phase separation technique on porous architecture of scaffolds for bone tissue engineering. J Biomed Mater Res Part B Appl Biomater 102:1304-1315. doi:10.1002/jbm.b.33101

[18] Tamjid E, Bagheri R, Vossoughi M, Simchi A (2011) Effect of particle size on the in vitro bioactivity, hydrophilicity and mechanical properties of bioactive glass-reinforced polycaprolactone composites. Mater Sci Eng, C 31:1526-1533. doi:10.1016/j.msec.2011.06.013

[19] Woodruff MA, Hutmacher DW (2010) The return of a forgotten polymer-polycaprolactone in the 21 st century. Prog Polym Sci 35:1217-1256. doi:10.1016/j.progpolymsci.2010. 04.002

[20] Lam CXF, Savalani MM, Teoh S-H, Hutmacher DW (2008) Dynamics of in vitro polymer degradation of polycaprolactone-based scaffolds: accelerated versus simulated physiological conditions. Biomed Mater 3:34108. doi:10.1088/ 1748-6041/3/3/034108

[21] Dziadek M, Zagrajczuk B, Jelen P et al (2016) Structural variations of bioactive glasses obtained by different synthesis routes. Ceram Int 43:14700-14709. doi:10.1016/j.cer amint.2016.06.095

[22] Łączka M, Cholewa-Kowalska K, Osyczka AM (2016) Bioactivity and osteoinductivity of glasses and glassceramics and their material determinants. Ceram Int 42:14313-14325. doi:10.1016/j.ceramint.2016.06.077

[23] Cao W, Hench LL (1996) Bioactive materials. Ceram Int 22:493-507. doi:10.1016/0272-8842(95)00126-3

[24] Mami M, Lucas-Girot A, Oudadesse H et al (2008) Investigation of the surface reactivity of a sol-gel derived glass in the ternary system $\mathrm{SiO}_{2}-\mathrm{CaO}-\mathrm{P}_{2} \mathrm{O}_{5}$. Appl Surf Sci 254:7386-7393. doi:10.1016/j.apsusc.2008.05.340

[25] Łączka M, Cholewa K, Łączka-Osyczka A (1997) Gelderived powders of $\mathrm{CaO}-\mathrm{P}_{2} \mathrm{O}_{5}-\mathrm{SiO}_{2}$ system as a starting material to production of bioactive ceramics. J Alloys Compd 248:42-51. doi:10.1016/S0925-8388(96)02648-5

[26] Kokubo T, Takadama H (2006) How useful is SBF in predicting in vivo bone bioactivity? Biomaterials 27:29072915. doi:10.1016/j.biomaterials.2006.01.017

[27] Zhang K, Wang Y, Hillmyer MA, Francis LF (2004) Processing and properties of porous poly(l-lactide)/bioactive glass composites. Biomaterials 25:2489-2500. doi:10.1016/ j.biomaterials.2003.09.033

[28] Lu L, Hou W, Sun J et al (2014) Preparation of poly (vinyl alcohol) fibers strengthened using multiwalled carbon nanotubes functionalized with tea polyphenols. J Mater Sci 49:3322-3330. doi:10.1007/s10853-014-8039-0 
[29] Izquierdo-Barba I, Arcos D, Sakamoto Y et al (2008) Highperformance mesoporous bioceramics mimicking bone mineralization. Chem Mater 20:3191-3198. doi:10.1021/ $\mathrm{cm} 800172 \mathrm{x}$

[30] Castro F, Ferreira AN, Rocha F et al (2012) Characterization of intermediate stages in the precipitation of hydroxyapatite at 37 1C. Chem Eng Sci 77:150-156. doi:10.1016/j.ces. 2012.01.058

[31] Caridade SG, Merino EG, Alves NM et al (2013) Chitosan membranes containing micro or nano-size bioactive glass particles: evolution of biomineralization followed by in situ dynamic mechanical analysis. J Mech Behav Biomed Mater 20:173-183. doi:10.1016/j.jmbbm.2012.11.012

[32] Poh PSP, Hutmacher DW, Stevens MM, Woodruff MA (2013) Fabrication and in vitro characterization of bioactive glass composite scaffolds for bone regeneration. Biofabrication 5:45005. doi:10.1088/1758-5082/5/4/045005

[33] Misra SK, Ansari T, Mohn D et al (2010) Effect of nanoparticulate bioactive glass particles on bioactivity and cytocompatibility of poly(3-hydroxybutyrate) composites. J R Soc Interface 7:453-465. doi:10.1098/rsif.2009.0255

[34] Wang B, Ji J, Li K (2016) Crystal nuclei templated nanostructured membranes prepared by solvent crystallization and polymer migration. Nat Commun 7:12804. doi:10.1038/ ncomms 12804

[35] Guillen GR, Pan Y, Li M, Hoek EMV (2011) Preparation and characterization of membranes formed by nonsolvent induced phase separation: a review. Ind Eng Chem Res 50:3798-3817. doi:10.1021/ie101928r

[36] Tang ZG, Black RA, Curran JM et al (2004) Surface properties and biocompatibility of solvent-cast poly[ $\varepsilon$-caprolactone] films. Biomaterials 25:4741-4748. doi:10.1016/j. biomaterials.2003.12.003

[37] Shikata T, Okuzono M (2013) Are all polar molecules hydrophilic? hydration numbers of ketones and esters in aqueous solution. J Phys Chem B 117:7718-7723. doi:10. 1021/jp4029968

[38] Smallwood IM (1996) 1,4-Dioxane. In: Handbook of organic solvent properties. Arnold, London, pp 213-215

[39] Gümüşderelioğlu M, Betül Kaya F, Beşkardeş IG (2011) Comparison of epithelial and fibroblastic cell behavior on nano/micro-topographic PCL membranes produced by crystallinity control. J Colloid Interface Sci 358:444-453. doi:10.1016/j.jcis.2011.03.026

[40] Ramiasa-MacGregor M, Mierczynska A, Sedev R, Vasilev K (2016) Tuning and predicting the wetting of nanoengineered material surface. Nanoscale 8:4635-4642. doi:10.1039/ C5NR08329J

[41] Ghaffarian V, Mousavi SM, Bahreini M, Afifi M (2013) Preparation and characterization of biodegradable blend membranes of PBS/CA. J Polym Environ 21:1150-1157. doi:10.1007/s10924-012-0551-1

[42] Dziadek M, Zagrajczuk B, Ziabka M et al (2016) The role of solvent type, size and chemical composition of bioactive glass particles in modulating material properties of poly(??caprolactone) based composites. Compos Part A Appl Sci Manuf 90:90-99. doi:10.1016/j.compositesa.2016.07.001

[43] Misra SK, Mohn D, Brunner TJ et al (2008) Comparison of nanoscale and microscale bioactive glass on the properties of $\mathrm{P}(3 \mathrm{HB}) /$ Bioglass $^{\circledR}$ composites. Biomaterials 29:1750-1761. doi:10.1016/j.biomaterials.2007.12.040

[44] Liu J, Ren L, Wei Q et al (2012) Microstructure and properties of polycaprolactone/calcium sulfate particle and whisker composites. Polym Compos 33:501-508. doi:10. $1002 / p c .22171$

[45] El-Hadi A, Schnabel R, Straube E et al (2002) Correlation between degree of crystallinity, morphology, glass temperature, mechanical properties and biodegradation of poly (3hydroxyalkanoate) PHAs and their blends. Polym Test 21:665-674. doi:10.1016/S0142-9418(01)00142-8

[46] Rezwan K, Chen QZ, Blaker JJ, Boccaccini AR (2006) Biodegradable and bioactive porous polymer/inorganic composite scaffolds for bone tissue engineering. Biomaterials 27:3413-3431. doi:10.1016/j.biomaterials.2006.01.039

[47] Washburn NR, Yamada KM, Simon CG et al (2004) Highthroughput investigation of osteoblast response to polymer crystallinity: influence of nanometer-scale roughness on proliferation. Biomaterials 25:1215-1224. doi:10.1016/j.bio materials.2003.08.043

[48] Cui H, Sinko PJ (2012) The role of crystallinity on differential attachment/proliferation of osteoblasts and fibroblasts on poly (caprolactone-co-glycolide) polymeric surfaces. Front Mater Sci 6:47-59. doi:10.1007/s11706-012-0154-8

[49] Liu J, Lu X, Li J, Wu C (2014) Preparation and properties of poly (vinylidene fluoride) membranes via the low temperature thermally induced phase separation method. J Polym Res 21:568. doi:10.1007/s10965-014-0568-3

[50] Salinas AJ, Vallet-Regí M (2013) Bioactive ceramics: from bone grafts to tissue engineering. RSC Adv 3:11116. doi:10. 1039/c3ra00166k

[51] Jaakkola T, Rich J, Tirri T et al (2004) In vitro Ca-P precipitation on biodegradable thermoplastic composite of poly (ع-caprolactone-co-dl-lactide) and bioactive glass (S53P4). Biomaterials 25:575-581. doi:10.1016/S0142-9612(03) 00558-1

[52] Poh PSP, Hutmacher DW, Stevens MM, Woodruff MA (2014) Corrigendum: fabrication and in vitro characterization of bioactive glass composite scaffolds for bone regeneration (2013 Biofabrication 5 045005). Biofabrication 6:29501. doi:10.1088/1758-5082/6/2/029501 
[53] Li W, Ding Y, Yu S et al (2015) Multifunctional chitosan45S5 bioactive glass-poly(3-hydroxybutyrate- co -3-hydroxyvalerate) microsphere composite membranes for guided tissue/bone regeneration. ACS Appl Mater Interfaces 7:20845-20854. doi:10.1021/acsami.5b06128

[54] Hench LL (2009) Genetic design of bioactive glass. J Eur Ceram Soc 29:1257-1265. doi:10.1016/j.jeurceramsoc.2008. 08.002

[55] Valerio P, Pereira MM, Goes AM, Leite MF (2004) The effect of ionic products from bioactive glass dissolution on osteoblast proliferation and collagen production. Biomaterials 25:2941-2948. doi:10.1016/j.biomaterials.2003.09.086

[56] Di Luca A, Ostrowska B, Lorenzo-Moldero I et al (2016) Gradients in pore size enhance the osteogenic differentiation of human mesenchymal stromal cells in three-dimensional scaffolds. Sci Rep 6:22898. doi:10.1038/srep22898

[57] Karageorgiou V, Kaplan D (2005) Porosity of 3D biomaterial scaffolds and osteogenesis. Biomaterials 26:5474-5491. doi:10.1016/j.biomaterials.2005.02.002 